\title{
COMPLETE TOPOLOGIES ON SPACES OF BAIRE MEASURE
}

\author{
BY
}

\author{
R. B. $\operatorname{KIRK}(1)$
}

ABSTRACT. Let $X$ be a completely regular Hausdorff space, let $L$ be the linear space of all finite linear combinations of the point measures on $X$ and let $M_{\sigma}$ denote the space of Baire measures on $X$. The following is proved: If $M_{\sigma}$ is endowed with the topology of uniform convergence on the uniformly bounded, equicontinuous subsets of $C^{b}(X)$, then $M_{\sigma}$ is a complete locally convex space in which $L$ is dense and whose dual is $C^{b}(X)$, provided there are no measurable cardinals. A complete description of the situation in the presence of measurable cardinals is also given. Let $M_{c}$ be the subspace of $M_{\sigma}$ consisting of those measures which have compact support in the realcompactification of $X$. The following result is proved: If $M_{c}$ is endowed with the topology of uniform convergence on the pointwise bounded and equicontinuous subsets of $C(X)$, then $M_{C}$ is a complete locally convex space in which $L$ is dense and whose dual is $C(X)$, provided there are no measurable cardinals. Again the situation if measurable cardinals exist is described completely. Let $M$ denote the Banach dual of $C^{b}(X)$. The following is proved: If $M$ is endowed with the topology of uniform convergence on the norm compact subsets of $C^{b}(X)$, then $M$ is a complete locally convex space in chich $L$ is dense. It is also proved that $M_{\sigma}$ is metrizable if and only if $X$ is discrete and that the metrizability of either $M_{c}$ or $M$ is equivalent to $X$ being finite. Finally the following is proved: If $M_{c}$ has the Mackey topology for the pair $\left(M_{c}, C(X)\right)$, then $M_{c}$ is complete and $L$ is dense in $M_{c}$.

Introduction. Throughout the paper, $X$ will denote a completely regular Hausdorff space (unless a statement is made to the contrary), $C$ or $C(X)$ will denote the linear space of all continuous real-valued functions on $X$ and $C^{b}$ or $C^{b}(X)$ will denote the subspace of $C$ consisting of those functions which are uniformly bounded. The linear space of all real-valued functions on $X$ which are zero on the complement of a finite subset will be denoted by $L$ or $L(X)$. (Hence $L$ is a realization of the free linear space generated by $X$ over the reals.) The space $L$ should be conceived as the space of all finite linear combinations of the point measures of unit mass on $X$. The spaces $L$ and $C$ may be paired in

Presented to the Society, August 30, 1972; received by the editors June 30, 1972 and, in revised form, January $28,1973$.

AMS (MOS) subject classifications (1970). Primary 60B10; Secondary 60B05.

Key words and phrases. Measures in topological spaces, $D$-spaces, equicontinuous families, complete locally convex topologies, approximation of measures.

(1) The author wishes to express his thanks to the Graduate School of Southern Illinois University for supporting released time in the summer of 1972 when this work was prepared. 
the sense of Bourbaki by the bilinear form defined by $\langle\xi, f\rangle=\Sigma\{\xi(x) f(x): x \in X\}$ for all $\xi \in L$ and $f \in C$. The same bilinear form defines by restriction a pairing of $L$ with $C^{b}$. Generally, the purpose of the paper will be to investigate the completion of $L$ with respect to several natural locally convex topologies on $L$ compatible with the pairs $(L, C)$ and $\left(L, C^{b}\right)$. The method used is to exploit systematically the well-known theorem of $A$. Grothendieck concerning complete locally convex spaces in the light of various results taken from the theory of measures in topological spaces. The net result will be to find complete spaces of Baire measures in which $L$ is a dense subspace.

The origin of the problem occurs in M. Katětov's papers [10] and [11]. Katětov's result can be described as follows. Identify $X$ with a subset of $L$ by the map $x m \rightarrow \delta_{x}$, where $\delta_{x}$ denotes the characteristic function of $\{x\}$. (Make this identification, and assume for the remainder of the paper that $X$ is a subset of $L_{0}$ ) Katětov considers a topology $e$ on $L$ which is the finest locally convex topology on $L$ whose restriction to $X$ is the original topology of $X$. He then proves that if $X$ is compact, then the completion of $(L, e)$ is the Banach dual of $C$. In [22] V. Ptăk extends this result to the case that $X$ is pseudocompact (Theorem 7, p. 187). In [23] D. A. Raikov characterizes the topology $e$ on $L$ as the topology of uniform convergence on the pointwise bounded, equicontinuous subsets of $C$ for the pair $(L, C)$. Since it is well known that if $X$ is pseudocompact, then the Banach dual of $C$ is the space of all finite signed Baire measures on $X$, the result of Katětov-Ptăk may be stated as follows:

Theorem. Let $X$ be pseudocompact. Then the completion of $L$ for the topology of uniform convergence on the pointwise bounded, equicontinuous subsets of $C$ is the space of all finite signed Baire measures on $X$.

The main results of the present paper are essentially generalizations of the above theorem to arbitrary completely regular spaces. (See Theorems $8.7,8.15$, 9.5, and 10.6.) Since if $X$ is not pseudocompact, then $C \neq C^{b}$, it is clear that more than one generalization is conceivable.

The paper itself has three major divisions. In part I, Baire measure on topological spaces, there are four sections. The purpose of this part is to establish the necessary background from the theory of measures in topological spaces. There are relatively few proofs, and the reader is referred for further details to the following : [8], [12], [13], [18], [19] and [25].

In part II, Topologies on the space $L$, there are three sections. In $\$ 5$ there are some preliminary results concerning equicontinuous families. In $\$ 6$ the topology $e$ is considered, and the improtant properties of this topology (obtained by Raikov in [23]) are established. In $\$ 7$ a topology $e^{b}$ analogous to $e$ is 
considered. Properties similar to those of $e$ are established for $e^{b}$. In particular, it is shown that $e^{b}$ is the finest locally convex topology on $L$ for which $X$ is a bounded subset and whose restriction to $X$ is the original topology on $X$ : Also in this section the topology $s$ on $L$ of uniform convergence on the norm compact sets in $C^{b}$ is investigated. A categorical property of this topology is established. This is that $s$ is the finest locally convex topology on $L$ for which $X$ is a totally bounded subset and whose restriction to $X$ is the original topology on $X$.

Part III, Completions of $L$, contains three sections. The main results of the paper are contained here. In $\$ 8$, it is shown that (except for some assumptions about measurable cardinals), the completion of $\left(L, e^{b}\right)$ is the space of all Baire measures on $X$. In $\$ 9$ it is shown that the completion of $(L, e)$ is the space of all Baire measures on $X$ which have compact support in the realcompactification of $X$. Also the completion of $(L, s)$ is shown to be the Banach dual of $C^{b}$. Finally in $\$ 10$ the Mackey topology for the pair $(L, C)$ is considered. The completion of $L$ with this topology is shown to be the space of all Baire measures with compact support if $X$ is realcompact.

\section{BAIRE MEASURES IN TOPOLOGICAL SPACES}

In order to make the paper self-contained, some facts from the the ory of measures in topological spaces will be reviewed here. The reader is referred to the works of Katětov [8]; Kirk [12], [13]; Knowles [16]; Moran [18], [19]; and Varadarajan [25].

1. The Banach dual of $C^{b}$. Let $\mathcal{Z}$ denote the family of zero sets in $X$ and let $\mathcal{F}$ denote the algebra of subsets of $X$ generated by $\mathcal{Z}$. The Baire algebra of $X$ is the $\sigma$-algebra generated by $Z$, or, equivalently, the smallest $\sigma$-algebra with respect to which the functions in $C$ (or $C^{b}$ ) are measurable. The Borel algebra of $X$ is the $\sigma$-algebra generated by the closed sets in $X$. The Borel algebra always contains the Baire algebra, but in general they are not equal.

Definition 1.1. A finitely-additive real-valued set function $m$ on $\mathcal{F}$ is regular if for each set $W \in \mathcal{F}$ and for each positive number $\epsilon$, there is a $Z \in \mathcal{Z}$ such that $Z \subset W$ and such that $\left|m\left(W^{\prime}\right)\right| \leq \epsilon$ whenever $W^{\prime} \in \mathcal{F}$ and $W^{\prime} \subset W-Z$.

The definitions of a regular set function on the Baire algebra and on the Borel algebra are essentially the same, except that in the case of the Borel algebra, one replaces $\mathcal{Z}$ with the family of closed sets in $X$. Let $M$ denote the space of all finitely-additive, real-valued regular set functions $m$ on $\mathcal{F}$ with the property that $\sup \{|m(W)|: W \in \mathcal{F}\}<\infty$. It can be shown (see [14]) that, with the usual pointwise definitions of addition, scalar multiplication and order, $M$ is an $L$-space in the sense of Kakutani. The following is due to A. D. Alexandrov [1]. (See [14] for related results.) 
Theorem 1.2 The Banach dual of $C^{b}$ is isomorphic as on L-space to M. The isomorphism may be realized by $\phi \leftrightarrow m$ where $\phi(f)=\int_{X} f d m$ for all $f \in C^{b}$. (The integral is the Riemann integral.)

For each functional $\phi$ in the Banach dual of $C^{b}$ (for each $m \in M$ ), denote by $\phi^{+}, \phi^{-}$and $|\phi|$ (by $\mathrm{m}^{+}, \mathrm{m}^{-}$and $|m|$ ) the positive, negative and total variation of $\phi$ (of $m$ ) respectively. The following result will be useful below. (See [14].)

Proposition 1.3. Let $m \in M$. For every positive number $\epsilon$, there are disjoint zero sets $Z_{1}$ and $Z_{2}$ such that $m^{+}\left(X-Z_{1}\right) \leq \epsilon$ and $m^{-}\left(X-Z_{2}\right) \leq \epsilon$.

Definition 1.4. Let $\phi$ belong to the Banach dual of $C^{b}$. Then $\phi$ is $\sigma$-additive if for every decreasing sequence $\left\{f_{n}\right\} \subset C^{b}$ with $f_{n} \downarrow 0$ pointwise, it follows that $|\phi|\left(f_{n}\right) \rightarrow 0$. The set function $m \in M$ is $\sigma$-additive if for every decreasing sequence of sets $\left\{Z_{n}\right\} \subset Z$ with $\bigcap\left\{Z_{n}: n \in N\right\}=\varnothing$, it follows that $|m|\left(Z_{n}\right) \rightarrow 0$.

The following definition gives an important continuity condition which is stronger than that of $\sigma$-additivity.

Definition 1.5. Let $\phi$ belong to the Banach dual of $C^{b}$. Then $\phi$ is netadditive if for every net $\left\{f_{i}\right\} \subset C^{b}$ which is directed downward to zero, it follows that $|\phi|\left(f_{i}\right) \rightarrow 0$. (The net $\left\{f_{i}\right\}$ is directed downward to zero if given $f_{i}$ and $f_{j}$, then there is $f_{k}$ such that $f_{k} \leq \inf \left(f_{i^{\prime}} f_{j}\right)$, and if $\inf \left\{f_{i}(x)\right\}=0$ for all $\left.x \in X_{0}\right)$ The set function $m \in M$ is net-additive if for every net $\left\{Z_{i}\right\} \subset Z$ which is directed downward to the empty set, it follows that $|m|\left(Z_{i}\right) \rightarrow 0$. (The net $\left\{Z_{i}\right\}$ is directed downward to the empty set if given $Z_{i}$ and $Z_{j}$, there is a $Z_{k}$ such that $Z_{k} \subset Z_{i}$ $\cap Z_{j}$ and if $\bigcap_{i}\left\{Z_{i}\right\}=\varnothing$.)

As might be expected, there is a relation between $\sigma$-additive and net-additive functionals and the corresponding types of set functions.

Proposition 1.6. Let $\phi$ belong to the Banach dual of $C^{b}$ and let $m \in M$ correspond to $\phi$ under the isomorphism of Theorem 1.2. Then the following bold:

(1) $\phi$ is $\sigma$-additive if and only if $m$ is $\sigma$-additive.

(2) $\phi$ is net-additive if and only if $m$ is net-additive.

We will use $M_{\sigma}$ and $M_{\tau}$ to denote the spaces of all $\sigma$-additive and all netadditive elements of $M$ respectively. These are ideals (which are even Dedekind complete) in the $L$-space $M_{\text {. }}$ Hence if $m \in M_{\sigma}\left(M_{\tau}\right)$, then $m^{+}, m^{-},|m| \in M_{\sigma}\left(M_{\tau}\right)$. It is easy to verify that if $m \in M_{\sigma}$ then $m$ is a countably-additive set function on $\mathcal{F}$. Hence by the Carathéodory process, every element in $M_{\sigma}$ has a uníque extension to a finite, regular signed Baire measure. For this reason we may identify $M_{\sigma}$ with the space of all finite, signed Baire measures. (Such measures are regular of necessity.)

The definition of a net-additive set function on the Borel algebra of $X$ is the 
same as Definition 1.5, except that zero sets are replaced by closed sets. The following is proved in [13].

Proposition 1.8. Let $m \in M$ be net-additive. Then there is a unique netadditive, regular signed Borel measure $\mu$ sucb that $\mu(W)=m(W)$ for all $W \in \mathcal{F}$.

In view of Proposition 1.8, it is not difficult to verify that $M_{\tau}$ may be identified $w$ ith the space of all net-additive, regular signed Borel measures.

\section{Supports.}

Definition 2.1. Let $m \in M$. Then the support of $m$ is defined by supp $m=$ $\bigcap\{Z: Z \in \mathcal{Z}$ and $|m|(Z)=|m|(X)\}$. (Note that supp $m$ is closed.)

It is easy to verify, since $M_{\tau}$ is an ideal in $M$, that supp $m$ is not empty whenever $0<m \in M_{\tau^{\circ}}$. However, in general, the support of $m$ may be empty even if $m \in M_{\sigma^{*}}$ (See [12].) A space $X$ in which every element of $M_{\sigma}$ has a nonempty support is called measure compact by Moran [18] and B-compact by Kirk [12]. It is shown by Pym in [21] that these are exactly the spaces in which $M_{\sigma}=M_{\tau^{*}}$. The following result is due to Katětov [8]. (See also Varadarajan [25].) First recall that a set $Y$ has a measurable cardinal if there is a probability measure defined on the $\sigma$-algebra of all subsets of $Y$ which is zero on all singleton sets. A set $Y$ has a nonmeasurable cardinal if it does not have a measurable cardinal. It is consistent with the axioms of set theory to assume that all sets have nonmeasurable cardinals (although it is not known whether or not this assumption is independent). See [4, pp. 164-166].) Furthermore, if the continuum hypothesis holds, then the continuum has a nonmeasurable cardinal.

Proposition 2.2. Let $X$ be paracompact and assume that every closed, discrete subspace of $X$ bas a nonmeasurable cardinal. Then $X$ is measure compact.

The following useful result is also proved by Varadarajan in [25].

Proposition 2.3. Let $X$ be paracompact and let $m \in M_{\tau^{*}}$. Then supp $m$ is a closed Lindelöf space.

3. The spaces $M_{s}$. Since we cannot guarantee that measurable cardinals do not exist, we must consider a subspace of $M_{\sigma}$ which was introduced by Dudley in [3] and which will play an important role later. We first recall some definitions.

A pseudometric on $X$ is a function $d$ from $X \times X$ into the real numbers which satisfies all the axioms of a metric except that $d(x, y)=0$ need not imply that $x=y$. The pseudometric $d$ is continuous if it a continuous function when $X \times X$ has the product topology, and it is bounded if there is a number $K$ such that $d(x, y) \leq K$ for all $x, y \in X$. If $d$ is a pseudometric on $X$, the relation $x \sim y$ if $d(x, y)=0$ is an equivalence relation on $X$. Let $X^{*}$ denote the set of 
equivalence classes and for $[x],[y] \in X^{*}$ define $d^{*}([x],[y])=d(x, y)$. Then $\left(X^{*}, d^{*}\right)$ is a metric space which is called the metric space associated with the pseudometric $d$.

Definition 3.1. Let $d$ be a continuous pseudometric on $X$. An element $m \in M_{\sigma}$ is called $d$-separable if there is a $d$-closed set $Z \subset X$ such that $|m|(X-Z)=0$ and such that $Z$ contains a countable $d$-dense subset.

Note that if $d$ is a continuous pseudometric on $X$ and if $Z \subset X$ is d-closed, then $Z \in \mathcal{Z}$. Indeed if $Z$ is not empty, then the function $f$ defined by $f(x)=$ $d(x, Z)=\inf \{d(x, y): y \in Z\}$ belongs to $C$ since $d$ is continuous, and $Z$ is the zero set of $f$ since $Z$ is $d$-closed. (Of course, if $Z=\varnothing$, then the result is clear.)

Definition 3.2. By $M_{s}$ denote the set of all those set functions $m \in M_{\sigma}$ such that $m$ is $d$-separable for every continuous pseudometric $d$ on $X$.

It is not hard to verify that $m \in M_{s}$ if and only if $m \in M_{\sigma}$ and $m$ is $d$ separable for every bounded continuous pseudometric on $X$. It is clear that if $m \in M_{s}$, then $m^{+}, m^{-},|m| \in M_{s}$ so that $M_{s}$ is an ideal in $M_{0}$. The following notion is due to Granirer [8].

Definition 3.3. The space $X$ is a $D$-space if whenever $d$ is a continuous pseudometric on $X$, then every $d$-discrete subspace of $X$ has a nonmeasurable cardinal.

The next result is essentially due to Marczewski and Sikorski [20], and it is also discussed by Dudley in [3].

Proposition 3.4. The space $X$ is a D-space if and only if $M_{s}=M_{\sigma}$

Since, as we have seen above, it is consistent to assume that all cardinals are nonmeasurable, it follows that it is consistent $w$ ith the axioms of set theory to assume that $M_{s}=M_{\sigma}$ is valid for all spaces $X$. Since metric spaces are, in particular, paracompact, a proof of Proposition 3.4 may be based on Proposition 2.2, Proposition 2.3 and the following lemma (which will be useful later).

Lemma 3.5. Let $d$ be a continuous pseudometric on $X$ and let $m \in M_{\sigma}$ Let $\Theta: X \rightarrow X^{*}$ be the canonical map of $X$ into the associated metric space. Then $\Theta^{-1}[W]$ is a Baire set (a zero set) in $X$ whenever $W$ is a Baire set (a closed set $)$ in $X^{*}$. Furthermore, if $m_{d}$ is defined by $m_{d}(W)=m\left(\Theta^{-1}[W]\right)$ for every Baire set $W$ in $X^{*}$, then $m_{d}$ is a regular, signed Baire measure on $X^{*}$.

Proof. Since $\Theta$ is continuous, it is clear that $\Theta^{-1}[W]$ is a Baire set in $X$ if $W$ is a Baire set in $X^{*}$. That $m_{d}$ is a regular, signed Baire measure on $X^{*}$ can be verified in a straightforward manner. It can be observed that if $W$ is closed in $X^{*}$, then $\Theta^{-1}[W]$ is a zero set in $X$ since $\Theta^{-1}[W]=\{x \in X: f(x)=0\}$ where $f(x)=\inf \left\{d(x, y): y \in \Theta^{-1}[W]\right\}$. The proof is complete. 
4. The order dual of $C$. In this section we will consider a representation theorem for the order dual of $C$ analogous to Theorem 1.2. Recall that a linear functional $\phi$ on the Riesz space $C$ is order bounded if, for every $0 \leq f \in C$, $\sup \{\phi(g): g \in C$ and $0 \leq g \leq f\}<\infty$. The set of all order bounded linear functionals on $C$ forms a Dedekind complete Riesz space called the order dual of $C$. The following result is due to Hewitt [7]. (Also see [5].) We will give a proof based on Theorem 1.2 which is shorter than Hewitt's original proof. (The proof that $\phi$ is an integral given below is due W. A. J. Luxemburg.)

Proposition 4.1. Let $\phi$ be a nonnegative linear functional on $C$. Then there is a regular Baire measure $m$ on $X$ such that $\phi(f)=\int_{X} f d m$ for all $f \in C$. Furthermore, if $X$ is realcompact, then $m$ bas compact support.

Proof. If $\phi$ is not an integral, then there is a decreasing sequence $\left\{f_{n}\right\} \subset C$ such that $f_{n} \downarrow 0$ pointwise on $X$ while $\phi\left(f_{n}\right) \geq 2 \alpha \phi(1)>0$ for all $n$, where $a$ is a fixed positive number. Define a function $f=\sum_{n=1}^{\infty}\left(f_{n}-\alpha\right)^{+}$. It is simple to verify that $f \in C$. However, for all $n$,

$$
\phi(f) \geq \sum_{k=1}^{n} \phi\left(\left(f_{k}-\alpha\right)^{+}\right) \geq \sum_{k=1}^{n}\left(\phi\left(f_{k}\right)-\alpha \phi(1)\right) \geq n \alpha \phi(1) .
$$

Hence $\phi(f)=\infty$ which is a contradiction.

Since $\phi$ is nonnegative, its restriction to $C^{b}$ belongs to the Banach dual of $C^{b}$. Hence by Theorem 1.2, there is a nonnegative $m \in M$ such that $\phi(f)=$ $\int_{X} f d m$ for all $f \in C^{b}$. Furthermore, $m$ is a Baire measure by Proposition 1.6. If $0 \leq f \in C$, set $f_{n}=\inf (f, n)$. Then $\left\{f_{n}\right\} \subset C^{b}$ and $f_{n} \uparrow f$ pointwise on $X$. Hence by the monotone convergence the orem and the fact that $\phi$ is an integral, $\phi(f)=$ $\lim \phi\left(f_{n}\right)=\lim \int_{X} f_{n} d m=\int_{X} f d m$. By the linearity of $\phi$, it follows that $\phi(f)=$ $\int_{X} f d m$ for all $f \in C$.

Now assume that $X$ is realcompact. If $Y \subset X$, let $\bar{Y}$ denote the closure of $Y$ in $\beta X$ (the Stone-Čech compactification of $X$ ). The family of zero sets $\{Z \epsilon$ $\mathcal{Z}: m(Z)=m(X)\}$ is clearly closed under finite intersections. Hence the set $G=$ $\bigcap\{\bar{Z}: Z \in \mathcal{Z}$ and $m(Z)=m(X)\}$ is a nonempty compact subset of $\beta X$. Of course, we assume that $m \neq 0$. The proof will be complete by Definition 2.1 if we can show that $G \subset X$.

If $G$ is not a subset of $X$, let $x_{0} \in G-X$. Since $X$ is realcompact, there is a function $g \in C$ with the property that $\bar{g}\left(x_{0}\right)=+\infty$ (where $\bar{g}$ is the unique continuous function taking values in the extended real numbers obtained by extending $g$ to $\beta X$ ). Define $Z_{n}=\{x \in X: g(x) \geq n\}$. Then for each $n, Z_{n}$ is a nonempty zero set in $X$ and $Z_{n+1} \subset Z_{n}$. Furthermore, $m\left(Z_{n}\right)>0$ for all $n$. Indeed, if $m\left(Z_{n_{0}}\right)=0$, let $Z=\left\{x \in X: g(x) \leq n_{0}\right\}$. Then $m(\dot{Z})=m(X)$ so that 
$x_{0} \in \bar{Z}$ by the definition of $G$. But this means $\bar{g}\left(x_{0}\right) \leq n_{0}$, which is contrary to assumption.

Since $Z_{n} \downarrow \varnothing$, it follows that $m\left(Z_{n}\right) \downarrow 0$. Hence, by passing to a subsequence if necessary, we may assume that $m\left(Z_{n+1}\right)<m\left(Z_{n}\right)$ for all $n$. Let $a_{n}=$ $m\left(Z_{n}\right)$, and let $\left\{f_{n}\right\}$ be a sequence in $C$ such that $0 \leq f_{n}, f_{n}=\left(a_{3 n+1}-a_{3 n+2}\right)^{-1}$ on the set $W_{n}=\{x \in X: 3 n+1 \leq g(x) \leq 3 n+2\}$ and $f_{n}=0$ on $\{x \in X: g(x) \leq 3 n$ or $g(x) \geq 3(n+1)\}$. If $f$ is defined by $f=\sum_{n=1}^{\infty} f_{n}$, it is easily verified that $f \in C$.

We then have for each $n \in N$ that

$$
\begin{aligned}
\phi(f) & \geq \sum_{k=1}^{n} \phi\left(f_{k}\right) \geq \sum_{k=1}^{n} \int_{W_{k}} f_{k} d m \geq \sum_{k=1}^{n}\left(a_{3 k+1}-a_{3 k+2}\right)^{-1} m\left(W_{k}\right) \\
& \geq \sum_{k=1}^{n}\left(a_{3 k+1}-a_{3 k+2}\right)^{-1} m\left(Z_{3 k+1}-Z_{3 k+2}\right) \\
& \geq \sum_{k=1}^{n}\left(a_{3 k+1}-a_{3 k+2}\right)^{-1}\left(a_{3 k+1}-a_{3 k+2}\right) \geq n .
\end{aligned}
$$

Hence it follows that $\phi(f)=\infty$. This is a contradiction, and the proof is complete.

Definition 4.2. An element $m \in M$ is said to have compact support in $\nu X$ (the realcompactification of $X$-see $[4, p, 116]$ ) if there is a compact set $G$ in $\nu X$ such that $|m|(X-Z)=0$ for every zero set $Z$ in $\nu X$ such that $G \subset Z$. Such a compact set will be called a compact support for $m$ in $\nu X$. Let $M_{c}$ denote the subspace of $M$ consisting of all those elements in $M$ which have compact support in $\nu X$.

We can now prove the following analogue of Theorem 1.2.

The orem 4.3. The order dual of $C$ is isomorpbic as a Riesz space to $M_{c} \cdot$ The isomorphism may be realized by the map $\phi \leftrightarrow m$ where $\phi(f)=\int_{X} f d m$ (where the integral is a Lebesgue integral).

Proof. Let $\phi$ be in the order dual of $C$. Let $\phi=\phi^{+}-\phi^{-}$be the Jordan decomposition of $\phi$. Then by Proposition 4.1, there are $0 \leq m_{1}, m_{2} \in M_{\sigma}$ such that $\phi^{+}(f)=\int_{X} f d m$, and $\phi^{-}(f)=\int_{X} f d m_{2}$ for all $f \in C$. By considering the restriction of $\phi$ to $C^{b}$ and applying Theorem 1.2, it follows that the map $\phi \rightarrow m$ is a Riesz space isomorphism of the order dual of $C$ onto a subspace $M^{*}$ of $M_{\sigma}$. In order to see that $M_{c} \subset M^{*}$, fix $0 \leq m \in M_{c}$ and fix $0 \leq f \in C$. If $G$ is a compact support for $m$ in $\nu X$, let $Z \equiv\left\{X \in \nu X:|\bar{f}(x)| \leq\|\bar{f}\|_{G}\right\}$ where $\bar{f}$ is the continuous extension of $f$ to $\nu X$. Since $m(X-Z)=0$, it follows that $\int_{X} f d m=$ $\int_{X \cap Z} f d m \leq\|\bar{f}\|_{G^{m}}(X)$. Thus $\int_{X} f d m<\infty$ for all $0 \leq f \in C$. It is now immediate that $M_{c} \subset M^{*}$. All that remains to show is that $M_{c}=M^{*}$.

Hence let $m \in M^{*}$ and assume without loss of generality that $0 \leq m$. Let 
$\phi(f)=\int_{X} f d m$ for all $f \in C$. For each $f \in C$, let $\bar{f}$ denote the unique continuous extension of $f$ to $\nu X$. Then the functional $\bar{\phi}$ defined by $\bar{\phi}(\bar{f})=\phi(f)$ for all $f \in C$ is a nonnegative linear functional on $C(\nu X)$. Since $\nu X$ is realcompact, it follows by Proposition 4.1 that the Baire measure representing $\bar{\phi}$ has compact support in $\nu X$. Let $G$ be the compact set in $\nu X$ which supports this measure. We will show that $G$ is a compact support for $m$. Indeed, let $Z$ be any zero set in $\nu X$ with $G \subset Z$, and define $U=X-Z$. If $W$ is any zero set in $X$ such that $W \subset U$, then $W \cap(X \cap Z)=\varnothing$. Hence there is $g \in C^{b}$ such that $0 \leq \bar{g} \leq 1, \bar{g}$ $=1$ on $W$ and $\bar{g}=0$ on $Z\left[4, \mathrm{p} .86\right.$. We then have that $m(W) \leq \int_{X} g d m=\phi(g)=$ $\bar{\phi}(\bar{g})=0$. Since $m(W)=0$ for any zero set $W \subset U$, the regularity of $m$ implies that $m(U)=0$. The proof is complete.

\section{TOPOLOGIES ON THE SPACE $L$}

5. Equicontinuous families. As always $X$ is a completely regular Hausdorff space. A set $B C C$ is equicontinuous (sometimes called locally equicontinuous) if for every positive number $\epsilon$ and for every $x \in X$, there is a neighborhood $U$ of $x$ such that $|f(x)-f(y)| \leq \epsilon$ for all $y \in U$ and all $f \in B$. The set $B$ is pointwise bounded if $\sup \{|f(x)|: f \in B\}$ is finite for each $x \in X$. The set $B$ is uniformly bounded if $\sup \left\{\|f\|_{X}: f \in B\right\}$ is finite. We will let $\mathcal{E}$ denote the family of all pointwise bounded and equicontinuous subsets of $C$. Let $E^{b}$ denote the family of all the sets in $\mathcal{E}$ which are also uniformly bounded. If $B \in \mathcal{E}$, then define $d_{B}(x, y)=\sup \{|f(x)-f(y)|: f \in B\}$. It is not difficult to verify that the function $d_{B}$ so defined is a continuous pseudometric on $X$. It is called the pseudometric associated with $B$. Let $p_{B}$ be the Minkowski function for $B^{\circ}$ (where $B^{\circ}$ denotes the polar of $B$ relative to the pair $(L, C)$ ). It is not difficult to verify that $p_{B}(\xi)=\sup \{|\langle\xi, f\rangle|: f \in B\}$ for every $\xi \in L$. It is clear from this that $d_{B}(x, y)=$ $p_{B}(x-y)$ for all $x, y \in X$. The following is a consequence of this observation.

Proposition 5.1. Let $B \in \mathcal{E}$. Then $B^{\circ \circ} \in \mathcal{E}$, where $B^{\circ \circ}$ is the bipolar of $B$ for the pair $(L, C)$.

Proof. It is clear that $B^{\circ 0}$ is pointwise bounded. Let $d_{B}$ be the pseudometric associated with $B$, and let $p_{B}$ be the Minkowski functional of $B^{\circ}$. Then $f \in B^{\circ \circ}$ if and only if $|\langle\xi, f\rangle| \leq p_{B}(\xi)$ for all $\xi \in L$. In particular, if $f \in B^{\circ \circ}$ and $x, y \in X$, then $|f(x)-f(y)|=|\langle x-y, f\rangle| \leq p_{B}(x-y)=d_{B}(x, y)$. Since $d_{B}$ is continuous, if $\epsilon>0$ and $x \in X$ are given, there is an open neighborhood $U$ of $x$ such that $d_{B}(x, y) \leq \epsilon$ whenever $y \in U$. It thus follows that $|f(x)-f(y)|$ $\leq \epsilon$ for all $y \in U$ and all $f \in B^{\circ \circ}$. Hence $B^{\circ \circ}$ is equicontinuous as was to be shown.

Proposition 5.2 Let $B \in \mathcal{E}^{b}$. Then $B^{\circ \circ} \in \mathcal{E}^{b}$, where $B^{\circ \circ}$ is the bipolar of $B$ for the pair $\left(L, C^{b}\right)$. 
Proof. Since $B^{\circ \circ}$ is a subset of the bipolar of $B$ for the pair $(L, C), B^{\circ 0} \epsilon$ $\mathcal{E}$ by Proposition 5.1. Since $B$ is uniformly bounded, it follows that the associated pseudometric $d_{B}$ is bounded. Let $d_{B}(x, y) \leq K$ for all $x, y \in X$ and fix a point $x_{0} \in X$. Let $M$ be a number such that $\left|f\left(x_{0}\right)\right| \leq M$ for all $f \in B$. Then if $x \in X$ and $f \in B$, it follows that $|f(x)| \leq\left|f(x)-f\left(x_{0}\right)\right|+\left|f\left(x_{0}\right)\right| \leq d_{B}\left(x, x_{0}\right)+M \leq K+M$. Hence $B^{\circ \circ}$ is uniformly bounded.

If $B \in \mathcal{E}$ and $A \subset B$ let $f^{A}$ be the real-valued function defined by $f^{A}(x)=$ $\sup \{f(x): f \in A\}$; and let $f_{A}$ be the real-valued function defined by $f_{A}(x)=$ inf $\{f(x): f \in A\}$. In particular, if $A=\{f\}$ then $f^{A}=f_{A}=f$. Define $B^{*}=\left\{f^{A}: A \subset B\right\}$ and $B_{*}=\left\{f_{A}: A \subset B\right\}$. We then have the following.

Proposition 5.3。 Let $B \in \mathcal{E}\left(\mathcal{E}^{b}\right)$. Then $B^{*} \in \mathcal{E}\left(\mathcal{E}^{b}\right)$ and $B_{*} \in \mathcal{E}\left(\mathcal{E}^{b}\right)$.

Proof. We will show that $B^{*} \in \mathcal{E}\left(\mathscr{E}^{b}\right)$. It follows from the definition that $B^{*}$ is pointwise (uniformly) bounded. In order to prove that $B^{*}$ is equicontinuous, it is clearly sufficient to show that if $f \in B^{*}$, then $|f(x)-f(y)| \leq d_{B}(x, y)$ for all $x, y \in X$. First let $f_{1}, f_{2} \in B$, and let $f=\sup \left(f_{1}, f_{2}\right)$. Then for all $x, y \in X$, it is clear that

$$
|f(x)-f(y)| \leq \max \left\{\left|f_{1}(x)-f_{1}(y)\right|,\left|f_{2}(x)-f_{2}(y)\right|\right\} \leq d_{B}(x, y) .
$$

It then follows by induction that if $A$ is any finite subset of $B$, then $\mid f^{A}(x)$ $f^{A}(y) \mid \leq d_{B}(x, y)$ for all $x, y \in X$.

In general, let $A \subset B$ and $\epsilon>0$ be arbitrary. Fix $x, y \in X$. By the definition of $f^{A}$, there are $f_{1}, f_{2} \in A$ such that $0 \leq f^{A}(x)-f_{1}(x) \leq \epsilon / 2$ and $0 \leq f^{A}(y)-$ $f(y) \leq \epsilon / 2$. Let $f=\sup \left(f_{1}, f_{2}\right)$. It is then clear that $0 \leq f^{A}(x)-f(x) \leq \epsilon / 2$ and $0 \leq f^{A}(y)-f(y) \leq \epsilon / 2$. Hence we have that

$$
\left|f^{A}(x)-f^{A}(y)\right| \leq\left|f^{A}(x)-f(x)\right|+|f(x)-f(y)|+\left|f(y)-f^{A}(y)\right| \leq \epsilon+d_{B}(x, y) .
$$

Since $\epsilon>0$ was arbitrary, the result follows.

6. The topology $e$. Since $C \doteq \bigcup \mathscr{E}$ and since each set in $\mathscr{E}$ is $\sigma(C, L)$ bounded, it follows $[17$, p. 255] that the topology on $L$ of uniform convergence on the sets in $\mathcal{E}$ is a locally convex topology. This topology will be denoted by $e$. In this section we will consider some fundamental properties of the space $(L, e)$. The results are due to Raikov [23].

Proposition 6.1. The dual space of $(L, e)$ is $C$.

Proof. Since $e$ is finer than $\sigma(L, C)$, it is clear that $C$ is contained in the dual of $(L, e)$. Now let $\phi$ be an $e$-continuous linear functional on $L$, and let $f_{0}$ denote the restriction of $\phi$ to $X$. Since $\phi$ is e-continuous, it is uniformly bounded on $B^{\circ}$ for some $B \in \mathcal{E}$. Let $M$ be a bound. For $x \in X$ let $U$ be a 
neighborhood of $x$ such that, for all $y \in U$ and all $f \in B,|f(x)-f(y)| \leq \epsilon M^{-1}$. Then for all $y \in U, M \epsilon^{-1}(x-y) \dot{\epsilon} B^{\circ}$ so that $\left|f_{0}(x)-f_{0}(y)\right|=|\phi(x-y)| \leq M\left(\epsilon M^{-1}\right)$ $=\epsilon$. Hence $f_{0}$ is continuous. Since $\left\langle\xi, f_{0}\right\rangle=\phi(\xi)$ for all $\xi \in L$, the proof is complete.

The following proposition is easily verified.

Proposition 6.2. The restriction of $e$ to $X$ is the original topology of $X$.

Theorem 6.3. Let $E$ be a locally convex space and let $\psi: X \rightarrow E$ be continuous. Then there is a unique continuous linear transformation $T$ from $(L, e)$ into $E$ such that $T=\psi$ on $X$.

Proof. Let $T$ be the unique linear extension of $\psi$ to $L$, and let $V$ be an arbitrary convex, balanced, closed neighborhood of zero in $E$. Let $V^{\circ}$ be the polar of $V$ in the dual of $E$. Define $B=\left\{f \in R^{X}: \exists \phi \in V^{\circ}, f=\phi \circ \psi\right\}$. It is clear that $B \subset C(X)$. Also $B$ is pointwise bounded. Indeed, fix $x \in X$. Then for some $n \in N, n^{-1} \psi(x) \in V$ so that $|f(x)|=|\phi \circ \psi(x)| \leq n$ for all $f \in B$. Furthermore, $B$ is equicontinuous. Indeea, fix $x \in X$ and $\epsilon>0$. Then $U=\left\{\psi^{-1}[\psi(x)+\epsilon V]\right\} \cap X$ is a neighborhood of $x$. Also, if $y \in U$ and $f \in B$, then there is $\phi \epsilon V^{\circ}$ such that $f=\phi \circ \psi$; and hence $|f(x)-f(y)|=|\phi(\psi(x)-\psi(y))| \leq \epsilon$.

It now follows that $T\left[B^{\circ}\right] \subset V^{\circ \circ}$. Indeed, fix $\xi \in B^{\circ}$, and let $\phi \in V^{\circ}$ be arbitrary. Then $f=\phi \circ \psi \in B$ so that $|\langle\xi, f\rangle| \leq 1$. Hence

$$
\begin{aligned}
|\phi(T(\xi))| & =\left|\phi\left(\sum\{\xi(x) \psi(x): x \in X\}\right)\right| \\
& =\left|\sum\{\xi(x) \phi \circ \psi(x): x \in X\}\right|=|\langle\xi, f\rangle| \leq 1 .
\end{aligned}
$$

Since $V=V^{\circ 0}$ by the bipolar theorem, it follows that $T\left[B^{\circ}\right] \subset V$, and the proof is complete.

Corollary 6.4. The topology $e$ is the finest locally convex topology on $L$ whose restriction to $X$ is the original topology of $X$.

7. The topology $e^{b}$. Since $C^{b}=\bigcup \mathcal{E}^{b}$ and since every set in $\mathscr{E}^{b}$ is $\sigma\left(C^{b}, L\right)$-bounded, the topology of uniform convergence on the sets in $\mathcal{G}^{b}$ is a locally convex topology on $L$. This topology will be denoted by $e^{b}$. In this section, we will establish properties of $e^{b}$ analogous to those of $e$ above.

Proposition 7.1. The dual of $\left(L, e^{b}\right)$ is $C^{b}$.

Proof. Since $e^{b}$ is finer than $\sigma\left(L, C^{b}\right)$, it is clear that $C^{b}$ is a subset of the dual space. Now let $\phi$ be an $e^{b}$-continuous linear functional on $L$. Then $\phi$ is $e$-continuous so that by Proposition 6.1, there is an $f \in C$ such that 
$\phi(\xi)=\langle\xi, f\rangle$ for all $\xi \in L$. It must be shown that $f$ is uniformly bounded. Let $B \in \mathcal{E}^{b}$ be such that $\phi$ is bounded on $B^{\circ}$. Thus there is a number $M$ such that $|\langle\xi, f\rangle| \leq M$ for all $\xi \in B^{\circ}$. Let $K$ be a uniform bound for all of the functions in $B$. Then $K^{-1} x \in B^{\circ}$ for all $x \in X$ so that $|f(x)| \leq K \cdot M$ for all $x \in X$. The proof is complete.

Proposition 7.2. The restriction of $e^{b}$ to $X$ is the original topology of $X$.

Theorem 7.3. Let $E$ be a locally convex space and let $\psi: X \rightarrow E$ be continuous. If $\psi[X]$ is bounded, then there is a unique continuous linear trans. formation from $\left(L, e^{b}\right)$ into $E$ such that $T=\psi$ on $X$.

Proof. Let $T$ be the unique linear extension of $\psi$ to $L$. Let $V$ be a closed, balanced and convex neighborhood of zero in $E$, and let $V^{\circ}$ be the polar of $V$ in the dual of $E$. Now define $B=\left\{f \in R^{X}: \exists \phi \in V^{\circ}, f=\phi \circ \psi\right\}$. Since $\psi[X]$ is bounded in $E$, it follows that $B \subset C^{b}$. Just as in Proposition 6.3, it follows

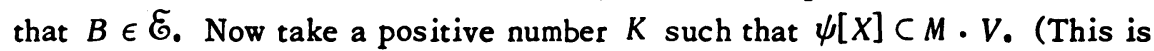
possible since $\psi[X]$ is bounded.) This means that $|f(x)|=|\phi(\psi(x))| \leq M$ for all $f \in B$. Hence $B \in \mathcal{E}^{b}$. Finally, as in the proof of Theorem $6.3, T\left[B^{\circ}\right] \subset V^{\circ 0}=V$.

Corollary 7.4. The topology $e^{b}$ is the finest locally convex topology on $L$ whose restriction to $X$ is the original topology of $X$ and for whicb $X$ is a bounded set.

Proof. In view of Theorem 7.3, it is sufficient to show that $X$ is bounded in $\left(L, e^{b}\right)$. But this is immediate since the restriction to $X$ of every continuous linear functional on $(L, e)$ is bounded by Proposition 7.1.

In the remainder of this section, we will discuss the topology on $L$ of uniform convergence on the norm compact subsets if $C^{b}$. This topology will be denoted by $s$. The basic properties of the $s$ topology are consequences of the following proposition.(2)

Proposition 7.5. The space $(L, s)$ is a dense subspace of $\left(L(\beta X), e^{b}(\beta X)\right)$.

Proof. If $B \subset C^{b}$, let $\bar{B}=\{\bar{f}: f \in B\}$ where $f$ is the continuous extension of $f$ to $\beta X$. By the Ascoli-Arzelá theorem, $B$ is norm compact if and only if $\bar{B} \epsilon e^{b}(\beta X)$. Hence $s$ is the restriction of $e^{b}(\beta X)$ to L. From Proposition 7.1, it is immediate that $L$ is dense in $\left(L(\beta X), e^{b}(\beta X)\right)$.

The following is an immediate consequence of Propositions 7.1 and 7.5.

(2) The author would like to thank the referee for formulating Proposition 7.5 and for indicating the simplifications it affords to studying the $s$ topology (in particular in the proofs of Theorems $7.8,8.14$ and 8.16.) 
Proposition 7.6. The dual space of $(L, s)$ is $C^{b}$.

Since $X$ is dense in $\beta X$, the following is immediate from Propositions 7.2 and 7.5.

Proposition 7.7. The restriction of $s$ to $X$ is the original topology of $X$.

Theorem 7.8. Let $E$ be a locally convex space and let $\psi: X \rightarrow E$ be continuous. If $\psi[X]$ is totally-bounded, then there is a unique continuous linear transformation $T$ from $(L, s)$ into $E$ such that $T=\psi$ on $X$.

Proof. Let $\hat{E}$ denote the completion of $E$. Then $\psi[X]$ is relatively compact in $\hat{E}$. Let $\psi: \beta X \rightarrow \hat{E}$ be the continuous extension of $\psi$ to $\beta X$. By Theorem 7.3 there is a continuous linear transformation $\hat{T}$ from $\left(L(\beta X), e^{b}(\beta X)\right)$ into $\hat{E}$ such that $\hat{T}=\hat{\psi}$ on $\beta X$. Let $T$ denote the restriction of $\hat{T}$ to $L$. By Proposition 7.5, $T$ is a continuous linear transformation of $(L, s)$ into $E$. The uniqueness of $T$ is immediate.

Corollary 7.9. The topology $s$ is the finest locally convex topology on $L$ for which $X$ is totally bounded.

Proof. Since $X$ is relatively compact in $\left(L(\beta X), e^{b}(\beta X)\right)$, it follows from Proposition 7.5 that $X$ is totally bounded in $(L, s)$. The result is now immediate from Theorem 7.8.

\section{COMPLETIONS OF $L$}

In this part of the paper, we will investigate the structure of the completion of the space $L$ under several different topologies. Each of the main completion results (Theorems $8.7,8.15$ and 9.5 ) in $\$ \$ 8$ and 9 are generalizations of the Katětov-Ptăk theorem. A crucial tool in these investigations is the following theorem of A. Grothendieck which we will refer to as the completion theorem. (See $[17$, p. 270].)

Theorem. Let $E$ be a locally convex Hausdorff space, let $E^{\prime}$ denote the dual space of $E$ and let $\mathcal{U}$ denote a basis for the neighborbood system at zero in $E$. The completion of $E$ is the space $\hat{E}$ of all linear functionals on $E^{\prime}$ which are $\sigma\left(E^{\prime}, E\right)$-continuous on $U^{\circ}$ for every $U \in \mathcal{U}$. The topology of $\hat{E}$ is that of uniform convergence on the sets in the family $\left\{U^{\circ}: U \in \mathcal{U}\right\}$.

8. The completion of $\left(L, e^{b}\right)$. Denote the completion of $\left(L, e^{b}\right)$ by $\hat{L}^{b}$. We then have the following.

Proposition 8.1. $\hat{L}^{b}$ is a subspace of the Banacb dual of $C^{b}$.

Proof. If $\phi \in \hat{L}^{b}$, it then follows by Proposition 7.1 and the completion 
theorem that $\phi$ is a linear functional on $C^{b}$. Let $\left\{f_{n}\right\}$ be a sequence in $C^{b}$ such that $f_{n} \rightarrow 0$ uniformly. Then $\left\{f_{n}\right\} \in \mathcal{E}^{b}$. Since the family $\mathcal{U}=\left\{B^{\circ}: B \in \mathcal{E}^{b}\right\}$ is a local basis at zero for $e^{b}$ and since $\left\{f_{n}\right\}$ is a sequence in $\left\{f_{n}\right\}^{\circ 0}$ with $f_{n} \rightarrow 0$ in the $\sigma\left(C^{b}, L\right)$-sense (i.e. pointwise), it follows from the completion theorem that $\phi\left(f_{n}\right) \rightarrow 0$. Hence $\phi$ is in the Banach dual of $C^{b}$. The proof is complete.

As a consequence of Proposition 8.1 and Theorem 1.2, we may identify $\hat{L}^{b}$ with a subspace of $M$. The set functions in this subspace have some rather interesting continuity properties (which are, in a sense, generalized dominated convergence and monotone convergence results) as the following proposition shows.

Proposition 8.2. Let $m$ belong to M. Then the following are equivalent:

1. $m \in \hat{L}^{b}$.

2. Let $\left\{f_{i}\right\} \in \mathcal{E}^{b}$ be a net such that $f_{i} \rightarrow f$ pointwise on $X$. Then $\int_{X} f_{i} d m$ $\rightarrow \int_{X} f d m$.

3. Let $\left\{f_{i}\right\} \in \mathcal{E}^{b}$ be a downward directed net with $f_{i} \perp f$ pointwise on $X$. Then $\int_{X} f_{i} d m \rightarrow \int_{X} f d m$.

4. Let $\left\{f_{i}\right\} \in \mathcal{E}^{b}$ be a downward directed net with $f_{i} \downarrow 0$ pointwise on $X$. Then $\int_{X} f_{i} d m \rightarrow 0$.

5. Let $\left\{f_{i}\right\} \in \mathcal{E}^{b}$ be a net such that $f_{i} \rightarrow f$ pointwise on $X$. Then $\int_{X} f_{i} d|m|$ $\rightarrow \int_{X} f d|m|$.

Proof. $(1 \Rightarrow 2)$ This is immediate from the completion theorem since $\left\{f_{i}\right\}^{\circ}$ is an $e^{b}$-neighborhood of zero and since $\left\{f_{i}\right\}^{\circ 0} \in E^{b}$ by Proposition 5.2.

$(2 \Rightarrow 1)$ Since $\left\{B^{\circ}: B \in \mathcal{E}^{b}\right\}$ is a local base at zero for $e^{b}$ and since $B^{\circ \circ}$ $\epsilon \mathcal{E}^{b}$ whenever $B \in \mathcal{E}^{b}$ by Proposition 5.2, the result is immediate from the completion theorem.

( $2 \Rightarrow 3)$ This is obvious.

$(3 \Rightarrow 4)$ This is obvious.

$(4 \Rightarrow 5)$ Let $\left\{f_{i}\right\} \in E^{b}$ be a net such that $f_{i} \rightarrow f$ pointwise. Without loss of generality, assume that $f=0$. For each $i$, define $g_{i}=\sup \left\{f_{j}: i \leq j\right\}$. Then $\left\{g_{i}\right\}$ $\epsilon \mathbb{E}^{b}$ by Proposition 5.3. Also $\left\{g_{i}\right\}$ is a downward directed net with $g_{i} \downarrow 0$ pointwise on $X$.

We will now show that $\int_{X} g_{i} d|m| \rightarrow 0$, or, equivalently, that $\int_{X} g_{i} d m^{+} \rightarrow 0$ and $\int_{X} g_{i} d m^{-} \rightarrow 0$. Since the argument is the same for both, we will only show $\int_{X} g_{i} d m^{+} \rightarrow 0$. If this is not so, by passing to a subnet if necessary, we may assume that there is a positive number $a$ such that $0<3 a \leq \int_{X} g_{i} d m^{+}$for all $i$.

Since $\left\{g_{i}\right\} \in \mathcal{E}^{b}$ there is a uniform bound $K$ for the family $\left\{g_{i}\right\}$. By Proposition 1.3, there are disjoint zero sets $Z_{1}$ and $Z_{2}$ in $X$ with $m^{+}\left(X-Z_{1}\right) \leq a K^{-1}$ and $m^{-}\left(X-Z_{2}\right) \leq a K^{-1}$. Let $g \in C^{b}$ be such that $0 \leq g \leq 1, Z_{1}=\{x \in X: g(x)=$ $1\}$ and $Z_{2}=\{x \in X: g(x)=0\}$. Then the set of functions $\left\{g g_{i}\right\}$ belongs to $\mathcal{E}^{b}$ 
as can easily be verified. Since $\left\{g g_{i}\right\}$ is a downward directed net with $g g_{i} \downarrow 0$ pointwise on $X$, it follows by assumption that $\int_{X} g g_{i} d m \rightarrow 0$. On the other hand, we have the following:

$$
\begin{aligned}
\int_{X} g g_{i} d m & =\int_{X} g g_{i} d m^{+}-\int_{X} g g_{i} d m^{-} \\
& =-\int_{X-Z_{1}}(i-g) g_{i} d m^{+}+\int_{X} g_{i} d m^{+}-\int_{X-Z_{2}} g g_{i} d m^{-} \\
& \geq-K m^{+}\left(X-Z_{1}\right)+3 \alpha-K m^{-}\left(X-Z_{2}\right) \\
& \geq-K\left(\alpha K^{-1}\right)+3 \alpha-K\left(\alpha K^{-1}\right) \geq a>0
\end{aligned}
$$

Since the above inequality holds for all $i$, it contradicts the fact that $\int_{X} g g_{i} d m$ $\rightarrow 0$. Hence $\int_{X} g_{i} d|m| \rightarrow 0$ as asserted.

In order to complete the proof consider the nets $\left\{f_{i}^{+}\right\}$and $\left\{f_{i}^{-}\right\}$. Each of these belongs to $\mathcal{E}^{b}$ by Proposition 5.3, and each converges pointwise to zero on $X$ since $f_{i} \rightarrow 0$ pointwise. If for each $i, g_{i}^{+}=\sup \left\{f_{j}^{+}: i \leq j\right\}$ and $g_{i}^{-}=\sup \left\{f_{j}^{-}: i \leq j\right\}$, then we have shown above that $\int_{X} g_{i}^{+} d|m| \rightarrow 0$ and $\int_{X} g_{i}^{-} d|m| \rightarrow 0$. Since $0 \leq$ $\int_{X} f_{i}^{+} d|m| \leq \int_{X} g_{i}^{+} d|m|$ and $0 \leq \int_{X} f_{i}^{-} d|m| \leq \int_{X} g_{i}^{-} d|m|$, it follows that $\int_{X} f_{i} d|m|=$ $\int_{X} f_{i}^{+} d|m|-\int_{X} f_{i}^{-} d|m| \rightarrow 0$. This is what was to be shown.

$(5 \Rightarrow 2)$ Let $\left\{f_{i}\right\} \in \mathcal{E}^{b}$ be a net such that $f_{i} \rightarrow f$ pointwise on $X$. Assume without loss of generality that $f=0$. Then by Proposition 5.2, $\left\{\left|f_{i}\right|\right\} \in \varepsilon^{b}$ is a net such that $\left|f_{i}\right| \rightarrow 0$ pointwise on $X$. Hence, applying the assumption,

$$
\left|\int_{X} f_{i} d m\right| \leq \int_{X}\left|f_{i}\right| d|m| \rightarrow 0 .
$$

The proof of the proposition is complete

Proposition 8.3. The space $\hat{L}^{b}$ is an ideal in the Riesz space $M$.

Proof. We first show that $\hat{L}^{b}$ is a sublattice. To do this it is sufficient to prove that if $m \in \hat{L}^{b}$, then $|m| \epsilon \hat{L}^{b}$. But if $m \in \hat{L}^{b}$, then $|m|$ satisfies condition 5 of Proposition 8.2. But then the implication $(2 \Rightarrow 1)$ in Proposition 8.2 shows that $|m| \epsilon \hat{L}^{b}$. In order to see that $\hat{L}^{b}$ is an ideal, let $m_{1}, m_{2} \in M$ be such that $\left|m_{1}\right| \leq\left|m_{2}\right|$ and $m_{2} \in \hat{L}^{b}$. Let $\left\{f_{i}\right\} \in \mathcal{E}^{b}$ be a downward directed net with $f_{i} \downarrow 0$ pointwise on $X$. Then $\int_{X} f_{i} d\left|m_{2}\right| \rightarrow 0$ by condition 5 of Proposition 8.2. Since $\left|\int_{X} f_{i} d m_{1}\right| \leq \int_{X} f_{i} d\left|m_{1}\right| \leq \int_{X} f_{i} d\left|m_{2}\right|$, it follows that $\int_{X} f_{i} d m_{1} \rightarrow 0$. Hence $m_{1} \in \mathcal{L}^{b}$ by the implication $(4 \Rightarrow 1)$ of Proposition 8.2. The proof is complete.

Proposition 8.4. The space $\hat{L}^{b}$ is a subspace of $M_{\sigma}$.

Proof. Let $m \in \hat{L}^{b}$. By Proposition 1.6, it is sufficient to prove that if 
$\left\{f_{n}\right\} \subset C^{b}$ is a decreasing sequence with $f_{n} \downarrow 0$ pointwise on $X$, then $\int_{X} f_{n} d m$ $\rightarrow 0$. But it is an easy exercise to verify that if $f_{n} \downarrow 0$ pointwise, then $\left\{f_{n}\right\} \in \mathcal{E}^{b}$. The result is now immediate from Proposition 8.2.

The following result gives a necessary and sufficient condition for a nonnegative element of $M_{\sigma}$ to belong to $M_{s}$. Other equivalent conditions are given by Sentilles and Wheeler in [24]. The proof given be low is similar to that outlined in their work. (The author would like to take this opportunity to thank Professor Sentilles for sending him a copy of this work while it was still in progress.)

Proposition 8.5. Let $0 \leq m \in M_{\sigma}$. Then $m \in M_{s}$ if and only if for every net $\left\{f_{i}\right\} \in \mathcal{E}^{b}$ with $f_{i} \rightarrow f$ pointwise on $X$, it follows that $\int_{X} f_{i} d m \rightarrow \int_{X} f d m$.

Proof. $(\Rightarrow)$ Let $\left\{f_{i}\right\} \in \mathcal{E}^{b}$ be such that $f_{i} \rightarrow f$ pointwise on $X$. Without loss of generality, assume that $f=0$. If it is not true that $\int_{X} f_{i} d m \rightarrow 0$, then, by passing to a subnet if necessary, there is a positive number $a$ such that $\int_{X} f_{i} d m$ $\geq a>0$ for all $i$. Define $d(x, y)=\sup _{i}\left|f_{i}(x)-f_{i}(y)\right|$ for all $x, y \in X$. Then $d$ is a continuous pseudometric on $X$. Since $m \in M_{s}$, there is a zero set $Z_{d}$ in $X$ such that $m\left(X-Z_{d}\right)=0, Z_{d}$ is $d$-closed and $Z_{d}$ contains a countable $d$-dense set $Y$. Since $Y$ is countable, there is a sequence $\left\{g_{n}\right\}$ in $\left\{f_{i}\right\}$ such that $g_{n}(y) \rightarrow 0$ as $n \rightarrow \infty$ for all $y \in Y$.

It now follows that $g_{n}(x) \rightarrow 0$ as $n \rightarrow \infty$ for all $x \in Z_{d}$. Indeed, fix $x \in Z_{d}$ and $\epsilon>0$. Take $y \in Y$ such that $d(x, y) \leq \epsilon / 2$. Choose a natural number $n_{0}$ such that $\left|g_{n}(y)\right| \leq \epsilon / 2$ whenever $n \geq n_{0}$. Then for $n \geq n_{0}$ it follows that $\left|g_{n}(x)\right| \leq$ $\left|g_{n}(y)\right|+\left|g_{n}(y)-g_{n}(x)\right| \leq \epsilon / 2+d(x, y) \leq \epsilon$. Since $x \in X$ and $\epsilon>0$ were arbitrary, $g_{n} \rightarrow 0$ pointwise on $Z_{d}$ as claimed. But $m\left(X-Z_{d}\right)=0$ so that $g_{n} \rightarrow 0$ pointwise almost everywhere on $X$ for the measure $m$. By the dominated convergence theorem it follows that $\int_{X} g_{n} d m \rightarrow 0$ as $n \rightarrow \infty$. But this contradicts the fact that $\int_{X} f_{i} d m \geq \alpha>0$ for all $i$.

$(\Leftarrow)$ Let $d$ be an arbitrary continuous pseudometric on $X$. Let $m_{d}$ be the set function induced by $m$ on the associated metric space $\left(X^{*}, d^{*}\right)$ according to Proposition 3.5. Since $m \in M_{\sigma}$ it follows by Proposition 3.5 that $m_{d}$ is a Baire measure. Since a metric space is in particular paracompact, if $m_{d}$ is net-additive, it then follows by Proposition 2.3 that $m_{d}$ is supported on a $d$-closed, Lindelöf subspace $Z^{*}$ of $X^{*}$. Also $Z^{*}$ is $d$-separable since any Lindelöf subspace of a metric space is separable. Then $Z=\Theta^{-1}\left[Z^{*}\right]$ is a zero set in $X$ which is $d$ closed and $d$-separable (where $\Theta$ is the map of Lemma 3.5) and which is such that $m(X-Z)=0$. Thus all that need be shown is that $m_{d}$ is net-additive on $X^{*}$ for every continuous pseudometric on $X$.

Assume that $m_{d}$ is not net-add itive for some continuous pseudometric on $X$. We will show that we may assume without loss of generality that $m_{d}$ has an empty 
support. Since $m_{d}$ is not net-additive (as is shown in Pym [21]), there is a nonzero Baire measure $\mu^{*}$ on $X^{*}$ with $0<\mu^{*} \leq m_{d}$ which has empty support. Let $E$ be the subspace of $C^{b}(X)$ consisting of those functions $f$ such that $f=f^{*} \circ \Theta$ for some $f^{*} \in C^{b}\left(X^{*}\right)$. Define a functional $\phi^{*}$ on $E$ by $\phi^{*}(f)=\int_{X^{*}} f^{*} d \mu^{*}$ for all $f \in E$. Define a seminorm $p$ on $C^{b}(X)$ by $p(f)=\int_{X} f^{+} d m$ for all $f \in C^{b}$. It is clear that $\phi^{*}(f) \leq p(f)$ for all $f \in E$. By the Hahn-Banach theorem, there is an extension $\phi$ of $\phi^{*}$ such that $\phi(f) \leq p(f)$ for all $f \in C^{b}$. It is simple to verify that $0 \leq \phi$. Let $0 \leq \mu \in M$ represent $\phi$ according to 1.2. Then for $0 \leq f \in C^{b}, \int_{X} f d \mu \leq \bar{p}(f)=$ $\int_{X} f d m$. That is $0 \leq \mu \leq m$. Since $m \in M_{\sigma o}$ it follows that $\mu \in M_{\sigma}$. Furthermore, if $\left\{f_{i}\right\} \in \tilde{G}^{b}$ is a net such that $f_{i} \rightarrow f$ pointwise on $X$, then $\left|\int_{X}\left(f-f_{i}\right) d \mu\right| \leq$ $\int_{X}\left|f-f_{i}\right| d \mu \leq \int_{X}\left|f-f_{i}\right| d m \rightarrow 0$. Finally, since $\phi$ extends $\phi^{*}, \mu_{d}=\mu^{*}$. Hence $\mu$ satisfies the same conditions (relative to the proof) as $m$, and in addition has the property that $\mu_{d}$ has empty support.

As shown above we may assume that $m_{d}$ is entirely without support. This means that for each point $\xi \in X^{*}$, there is an open set $U_{\xi}$ containing $\xi$ such that $m_{d}\left(U_{\xi}\right)=0$. Since $\left\{U_{\xi}: \xi \in X^{*}\right\}$ is an open cover of $X^{*}$, there is a partition of unity $\left\{f_{i}^{*}: i \in I\right\}$ subordinate to this cover. (Hence, in particular, $\int_{X^{*}} f_{i}^{*} d m_{d}=$ 0 for all $i \in I$.) Define $f_{i}=f_{i}^{*} \circ \Theta$, and let $\mathcal{S}$ denote the family of all finite subsets of $I$. For $\sigma \in \mathcal{S}$ define $g_{\sigma}=\Sigma\left\{f_{i}: i \in \sigma\right\}$. Then $\left\{g_{\sigma}\right\} \in \mathcal{E}^{b}$. (This is true since $\left\{f_{i}^{*}\right\}$ being a partition of unity implies that there is an open set about each point in $X$ in which all but a finite number of the functions $f_{i}$ are zero.) Furthermore $g_{\sigma} \rightarrow 1$ pointwise on $X$. Hence by assumption $\int_{X} g_{\sigma} d m \rightarrow m(X)>0$. However $\int_{X} g_{\sigma} d m=\Sigma\left\{\int_{X^{*}} f_{i}^{*} d m_{d}: i \in \sigma\right\}=0$ for all $\sigma \in \mathcal{S}$. This is a contradiction, and the proof is complete.

Theorem 8.6. The completion of $\left(L, e^{b}\right)$ is the space $M_{s}$ equipped with the topology of uniform convergence on the sets in $\mathcal{E}^{b}$.

Proof. We will begin by showing that $\hat{L}^{b}$ is contained in $M_{s}$. By Proposition 8.3 it is sufficient to prove that if $0 \leq m \in \dot{\epsilon} \hat{L}^{b}$, then $m \in M_{s}$. However, this is immediate from the implication $(1 \Rightarrow 2)$ of Proposition 8.2 and Proposition 8.5. Again, to show that $M_{s}$ is contained in $\hat{L}^{b}$ (since $M_{s}$ is a lattice), it is enough to show that if $0 \leq m \in M_{s}$, then $m \in \hat{L}^{b}$. But this follows from Proposition 8.5 and the implication $(2 \Rightarrow 1)$ of Proposition 8.2. Hence $\hat{L}^{b}=M_{s}$. It follows from the completion theorem and Proposition 5.2 that the topology on $M_{s}$ as the completion of $\left(L, e^{b}\right)$ is the topology of uniform convergence on the sets in $\xi^{b}$. The proof is complete.

If $X$ is a $D$-space (Definition 3.3), then $\hat{L}^{b}=M_{s}-M_{\sigma}$. As noted above, it is consistent with the axioms of set theory to assume that every space is a $D$. space. Hence it is consistent to assume that $\hat{L}^{b}=M_{\sigma}$ for every completely 
regular Hausdorff space $X$. The main facts are summarized in the following theorem.

Theorem 8.7. Let $X$ be a completely regular Hausdorff space (a D-space) and let $M_{s}\left(M_{\sigma}\right)$ be endowed with the topology of uniform convergence on the uniformly bounded and equicontinuous subsets of $C^{b}$. Then the following bold:

1. $M_{s}\left(M_{\sigma}\right)$ is complete.

2. $L$ is dense in $M_{s}\left(M_{\sigma}\right)$.

3. The dual space for $M_{s}\left(M_{\sigma}\right)$ is $C^{b}$.

4. $X$ is a bounded subset of $M_{s}\left(M_{\sigma}\right)$.

5. The restriction of the topology to $X$ is the original topology of $X$.

Proof. Both 1 and 2 are immediate from Theorem 8.6. That 3 holds follows from Proposition 7.1 and the fact that the completion of a locally convex space has the same dual as the original space. Numbers 4 and 5 are just restatements of Proposition 7.2 and Corollary 7.4.

The following result was proved by Granirer in [6].

Corollary 8.8. Let $X$ be a completely regular Hausdorff space (a D-space).

1. If $B$ is a uniformly bounded and equicontinuous subset of $C^{b}$, then $B$ is relatively $\sigma\left(C^{b}, M_{s}\right)$-compact $\left(\sigma\left(C^{b}, M_{\sigma}\right)\right.$-compact $)$.

2. If $X$ is not a D-space, then there is a uniformly bounded and equicontinuous family in $C^{b}$ which is not relatively $\sigma\left(C^{b}, M_{\sigma}\right)$-compact.

Proof. (1) This is immediate from statement 3 of Theorem 8.7 and the Banach-Alaoglu theorem.

(2) If $X$ is not a $D$-space, $M_{s} \neq M_{\sigma}$. Let $M_{\sigma}$ have the topology of uniform convergence on the sets in $\xi^{b}$. By Theorem $8.7 M_{s}$ is a closed subspace of $M_{\sigma}$. Hence by the Hahn-Banach theorem, there is a nonzero continuous linear functional on $M_{\sigma}$ which vanishes on $M_{s}$. This means that the dual space of $M_{\sigma}$ is strictly larger than $C^{b}$. The result is now immediate from the Mackey-Arens the orem.

Remarks. 1. If $X$ is compact (or pseudocompact), then Theorem 8.6 reduces to the Katětov-Ptăk theorem.

2. If $X$ is discrete, then $\mathcal{E}^{b}$ is coextensive $w$ ith the family of uniformly bounded sets of bounded, real-valued functions on $X$. In the case, $M_{s}=l^{1}(X)$ with its norm topology.

In view of Remark 2, it is natural to ask if there are any other cases in which $M_{s}$ is normable. The following provides a negative answer to the question.

Theorem 8.9. Let $X$ be a completely regular Hausdorff space, and let $M_{s}$ bave the topology of uniform convergence on the uniformly bounded, equicontinuous subsets of $C^{b}$. Then the following are equivalent: 
1. $X$ is discrete.

2. $M_{s}$ is normable.

3. $M_{s}$ is metrizable.

4. $\left(L, e^{b}\right)$ is a Mackey space. (That is, the topology $e^{b}$ on $L$ is the Mackey topology for the pair $\left(L, C^{b}\right)$.)

Proof. $(1 \Rightarrow 2)$ This is the content of Remark 2 above.

$(2 \Rightarrow 3)$ This is obvious.

$(3-4)$ This is a consequence of the fact that any metrizable locally convex space is a Mackey space.

$(4 \Rightarrow 1)$ Let $m\left(L, C^{b}\right)$ and $m\left(M, C^{b}\right)$ denote the Mackey topologies for the pairs $\left(L, C^{b}\right)$ and $\left(M, C^{b}\right)$ respectively. Let $r$ and $\sigma$ denote the restrictions to $L$ of the topologies $m\left(M, C^{b}\right)$ and $\sigma\left(M, C^{b}\right)$ respectively. Since $L \subset M$, it is not difficult to verify that $\left(^{*}\right) m\left(L, C^{b}\right) \supset r \supset \sigma$. By assumption $m\left(L, C^{b}\right)$ is the topology of uniform convergence on the sets in $\xi^{b}$ so that by Theorem $8.7(5)$, the restriction of $m\left(L_{t} C^{b}\right)$ to $X$ is the original topology of $X$. Since it is well known that the restriction to $X$ of $\sigma\left(M, C^{b}\right)$ is the original topology of $X$, it follows that the restriction of $m\left(M, C^{b}\right)$ to $X$ is the original topology of $X$. However, in [15] it is shown that the restriction of $m\left(M, C^{b}\right)$ to $X$ is the original topology of $X$ if and only if $X$ is discrete. The proof is complete.

We will now discuss the question of when $M_{\sigma}^{+}=\left\{m \in M_{\sigma}: 0 \leq m\right\}$ is metrizable for the topology of uniform convergence on the sets in $\mathscr{G}^{b}$. For this purpose we recall two important theorems from the literature. The first is due to Granirer in [6].

Theorem 8.10. Let $X$ be a D-space. If $\left\{m_{i}\right\} \subset M_{\sigma}^{+}$is a net and if $\left\{m_{i}\right\}$ converges to $m \in M_{\sigma}^{+}$in the $\sigma\left(M_{\sigma}, C^{b}\right)$-sense, then $m_{i} \rightarrow m$ for the topology of uniform convergence on the uniformly bounded and equicontinuous subsets of $C^{b}$.

Note that Theorem 8.10 implies that if $X$ is a $D$-space, then on $M_{\sigma}^{+}$the topology of uniform convergence on the sets $E^{b}$ is identical with $\sigma\left(M_{\sigma}, C^{b}\right)$. The next theorem is due to Varadarajan in [25].

Theorem 8.11. Let $(X, d)$ be a metric space. Then $M_{\tau}^{+}=\left\{m \in M_{\tau}: 0 \leq m\right\}$ is metrizable for the weak topology $\sigma\left(M_{r}, C^{b}\right)$.

We can now prove the following.

Theorem 8.12. Let $X$ be a D-space. Then $X$ is metrizable if and only if $M_{\sigma}^{+}$is metrizable for the topology of uniform convergence on the uniformly bounded and equicontinuous sets in $C^{b}$.

Proof. Since $X \subset M_{\sigma}^{+}$it follows from condition (5) of Theorem 8.7 that if 
$M_{\sigma}^{+}$is metrizable, then $X$ is also metrizable. Now assume that $X$ is metrizable. Then by Proposition 2.2, $M_{\tau}=M_{\sigma}$. By Varadarajan's result (Theorem 9.11), the topology $\sigma\left(M_{\sigma}, C^{b}\right)$ on $M_{\sigma}^{+}$is metrizable. By Granirer's result (Theorem 8.10), the topology of uniform convergence on the sets in $\mathcal{E}^{b}$ is identical with $\sigma\left(M_{\sigma}, C^{b}\right)$ on $M_{\sigma}^{+}$Hence the result follows.

Finally we include the following result due to Dudley [3].

Theorem 8.13. Let $X$ be completely regular Hausdorff, and let $M_{s}$ be endowed with the topology of uniform convergence on the pointwise bounded and equicon. tinuous sets in $C^{b}$. A set $A \subset M_{s}$ is relatively sequentially compact if and only if it is weakly relatively sequentially compact.

Proof. In [3], Dudley proves that a sequence $\left\{m_{n}\right\} \subset M_{s}$ converges weakly to $m \in M_{s}$ if and only if it converges uniformly on the sets in $\mathcal{E}^{b}$. The result is the immediate.

The remainder of this section will be devoted to a discussion of the space $(L, s)$.

Theorem 8.14. The completion of $(L, s)$ is the space $M$ equipped with the topology of uniform convergence on the norm compact subsets of $C^{b}$.

Proof. By The orem 8.6 the completion of $\left(L(\beta X), e^{b}(\beta X)\right)$ is $M_{s}(\beta X)=$ $M(X)$. But $(L, s)$ is dense in $\left(L(\beta X), e^{b}(\beta X)\right)$ so that the result follows immediately.

The basic results about the $s$ topology are summarized in the following.

Theorem 8.15. Let $X$ be a completely regular Hausdorff space and let $M$ be equipped with the topology of uniform convergence on the norm compact subsets of $C^{b}$. Then the following bold:

1. $M$ is complete.

2. $L$ is dense in $M$.

3. The dual space of $M$ is $C^{b}$.

4. $X$ is a totally bounded subset of $M$.

5. The restriction of the topology on $M$ to $X$ is the original topology of $X$.

Remark. If $X$ is compact, then Theorem 8.15 reduces to the Katětov-Ptăk theorem via the Ascoli-Arzelá theorem.

Theorem 8.16. Let $X$ be a completely regular Hausdorff space and let $M$ be endowed with the topology of uniform convergence on the norm compact subsets of $C^{b}$. Then the following are equivalent:

1. $X$ is finite.

2. $M$ is normable.

3. $M$ is metrizable. 
Proof. We only need to show that 3 implies 1. But from Proposition 7.5, if $M$ is metrizable, then $M_{s}(\beta X)$ is metrizable (for the topology of uniform convergence on the uniformly bounded, equicontinuous subsets of $C(\beta X)$ ): Hence by Theorem 8.9, $\beta X$ is discrete. But this implies that $X$ is finite.

9. The completion of $(L, e)$. Let $\hat{L}$ denote the completion of $(L, e)$. We then have the following.

Proposition 9.1. $\mathcal{L}$ is a subspace of the order dual of $C$.

Proof. If $\phi \in \hat{L}$, then it follows from Proposition 6.1 and the completion theorem that $\phi$ is a linear functional on $C$. If $\phi$ does not belong to the order dual of $C$, then there is a function $f \in C$ with $0 \leq f$ and a sequence $\left\{g_{n}\right\} \subset C$ such that $0 \leq g_{n} \leq f$ and such that $0<\left|\phi\left(g_{n}\right)\right|=\alpha_{n} \uparrow+\infty$. Now define $b_{n}=$ $a_{n}^{-1 / 2} g_{n}$ for all $n \in N$. Then $b_{n} \rightarrow 0$ in the $\sigma(C, L)^{n}$-sense (i.e. pointwise), and $\left\{b_{n}\right\}$ is pointwise bounded. Furthermore, $\left\{b_{n}\right\}$ is equicontinuous. Indeed, let $x \in X$ and let $\epsilon$ be a positive number. Choose $n_{0} \in N$ such that $\alpha_{n}^{-1 / 2} \leq$ $\epsilon(\epsilon+f(x))^{-1}$ for all $n \geq n_{0}$. Let $U$ be a neighborhood of $x$ such that if $y \in U$, then (1) $|f(x)-f(y)| \leq \epsilon$ and (2) $\left|b_{n}(x)-b_{n}(y)\right| \leq \epsilon$ for $n=1,2, \ldots, n_{0}$. Now observe, since $0 \leq g_{n} \leq f$ for all $n \in N$, that $\left|g_{n}(x)-g_{n}(y)\right| \leq \epsilon+f(x)$ for all $y \in U$ by (1). Hence if $n \geq n_{0}$, we have for all $y \in U$ that $\left|b_{n}(x)-b_{n}(y)\right|=$ $a_{n}^{-1 / 2}\left|g_{n}(x)-g_{n}(y)\right| \leq \epsilon(\epsilon+f(x))^{-1}(\epsilon+f(x))=\epsilon$. On the other hand, if $n=1$, $\cdots, n_{0}$, then for all $y \in U$ we have by 2 that $\left|b_{n}(x)-b_{n}(y)\right| \leq \epsilon$. Thus we have proved that $\left\{b_{n}\right\} \in \mathcal{E}$. Since $\phi \in \hat{L}$ and since $b_{n} \rightarrow 0$ pointwise, it follows from the completion theorem that $\phi\left(b_{n}\right) \rightarrow 0$. However, $\left|\phi\left(b_{n}\right)\right|=a_{n}^{-1 / 2}\left|\phi\left(g_{n}\right)\right|=$ $\alpha_{n}^{1 / 2} \nearrow+\infty$. This is a contradiction, and the proof is complete.

As a consequence of Theorem 4.4 and Proposition 9.1 we may identify $\hat{L}$ with a subspace of $M_{c}$. The following gives some more information about this subspace.

Proposition 9.2. $\hat{L}$ is a subspace of $M_{s^{\circ}}$.

Proof. If $\phi \in \hat{L}$, it then follows by Proposition 9.1 that $\phi$ is a linear functional on $C$. Since $\left\{B^{\circ}: B \in \mathcal{E}\right\}$ is a local base at zero for $e$, it follows by the completion theorem and by Proposition 5.1 that $\phi$ is $\sigma(C, L)$-continuous on each $B \in \mathcal{E}$. In particular, the restriction of $\phi$ to $C^{b}$ is $\sigma\left(C^{b}, L\right)$-continuous on each $B \in E^{b}$ so that the restriction of $\phi$ to $C^{b}$ belongs to the completion of $\left(L, e^{b}\right)$ by the completion theorem. Hence by Theorem 8.6, there is an $m \in M_{s}$ such that $\phi(f)=\int_{X} f d m$ for all $f \in C^{b}$. But then by Proposition 9.1 and Theorem 4.4, it is immediate that $m$ is the element in $M_{c}$ which represents $\phi$ on $C$. The proof is complete. 
Proposition 9.3. Let $0 \leq m \in M$. Then the following are equivalent:

1. $m \in \hat{L}$.

2. If $\left\{f_{i}\right\} \in \mathcal{G}$ is a net with $f_{i} \rightarrow f$ pointwise on $X$, then $\int_{X} f_{i} d m \rightarrow \int_{X} f d m$.

3. If $\left\{f_{i}\right\} \in \mathcal{E}$ is a downward directed net with $f_{i} \downarrow f$ pointwise on $X$, then $\int_{X} f_{i} d m \rightarrow \int_{X} f d m$.

4. If $\left\{f_{i}\right\} \in \mathcal{E}$ is a downward directed net with $f_{i} \downarrow 0$, then $\int_{X} f_{i} d m \rightarrow 0$.

Proof. The proof of the implications $(1 \Leftrightarrow 2 \Rightarrow 3 \Rightarrow 4)$ is just as in Proposition 8.2.

$(4 \Rightarrow 2)$ Let $\left\{f_{i}\right\} \in \mathcal{E}$ be a net with $f_{i} \rightarrow f$ pointwise on $X$ and as sume $f=0$. By Proposition 5.3 we may assume $0 \leq f_{i}$ for all $i$. (Otherwise consider $\left\{f_{i}^{+}\right\}$ and $\left\{f_{i}^{-}\right\}$separately.) For each $i$ define $g_{i}=\sup \left\{f_{j}: i \leq j\right\}$. Then by Proposition 5.3, $\left\{g_{i}\right\} \in \mathcal{E}$ is a downward directed net with $g_{i} \downarrow 0$. Hence by assumption $\int_{X} g_{i} d m \rightarrow 0$. Since $0 \leq \int_{X} f_{i} d m \leq \int_{X} g_{i} d m$ for all $i$, the proof is complete.

We will now represent the completion of the space $(L, e)$. For this purpose, define $M_{s c}=M_{c} \cap M_{s}$. Then $M_{s c}$ is an ideal in the Riesz space $M$.

Theorem 9.4. The completion of the space $(L, e)$ is the space $M_{\text {sc }}$ equipped with the topology of uniform convergence on the sets in $\mathcal{E}_{\text {. }}$

Proof. It is a consequence of Proposition 9.1, Proposition 9.2 and Theorem 4.4 that $\hat{L}$ is a subspace of $M_{s c}$. In order to prove the reverse inclusion, take $m \in M_{s c}$, and assume $w$ ithout loss of generality that $0 \leq m$. Since $\left\{B^{\circ}: B \in \mathcal{E}\right\}$ is a local base at zero for $e$, it is sufficient by Proposition 9.3 to prove that if $\left\{f_{i}\right\} \in \mathcal{G}$ is downward directed net with $f_{i} \downarrow 0$ pointwise on $X$, then $\int_{X} f_{i} d m \rightarrow$ 0 . For each $f \in C$, let $\bar{f}$ denote the unique continuous extension of $f$ to $\nu X$. Define a nonnegative functional $\bar{\phi}$ on $C(\nu X)$ by $\bar{\phi}(\bar{f})=\int_{X} f d m$ for all $f \in C$. (The definition makes sense by Proposition 4.3(b).) Then by Theorem 4.4, there is a regular Baire measure $\bar{m}$ on. $\nu X$ which has a compact set $G \subset \nu X$ as its support and which is such that $\bar{\phi}(\bar{f})=\int_{\nu X} \bar{f} d \bar{m}$ for all $f \in C$. If we can show that $\bar{f}_{i} \downarrow 0$ pointwise on $G$, it will follow by Dini's theorem that $\bar{f}_{i} \downarrow 0$ uniformly on $G$. Hence $\phi\left(f_{i}\right)=\bar{\phi}\left(\bar{f}_{i}\right)=\int_{\nu X} \bar{f}_{i} d \bar{m}=\int_{G} \bar{f}_{i} d \bar{m} \rightarrow 0$. (Since $\bar{m}$ has compact support, it is net-additive and so a Borel measure by Proposition 1.8. Hence $\int_{G} \bar{f}_{i} d \bar{m}$ makes sense.)

We will now show that $\bar{f}_{i} \downarrow 0$ pointwise on $G$. Define $d(x, y)=$ $\sup \left\{\left|f_{i}(x)-f_{i}(y)\right|\right\}$. Then $d$ is a continuous pseudometric on $X$. Since $m \in M_{s}$, there is a $d$-closed zero set $Z_{d}$ in $X$ such that $m\left(X-Z_{d}\right)=0$ and a countable, $d$-dense subset $Y$ in $Z_{d}$. Let $\left\{g_{n}\right\}$ be a decreasing sequence in $\left\{f_{i}\right\}$ with $g_{n}$ $\downarrow 0$ pointwise on $Y$. Then $g_{n} \downarrow 0$ pointwise on $Z_{d}$. Indeed, take $x \in Z_{d}$ and $\epsilon>0$. Choose $y \in Y$ with $d(x, y) \leq \epsilon / 2$. Then take $n_{0}$ so large that for 
$n \geq n_{0}, g_{n}(y) \leq \epsilon / 2$. Hence for $n \geq n_{0},\left|g_{n}(x)\right| \leq\left|g_{n}(x)-g_{n}(y)\right|+\left|g_{n}(y)\right| \leq d(x, y)$ $+\epsilon / 2 \leq \epsilon$. Thus $g_{n} \downarrow 0$ on $Z_{d}$ as asserted.

Let $Z^{*}$ be any zero set in $\nu X$ with $X \cap Z^{*}=Z_{d}$. Then $G \subset Z^{*}$. Indeed, let $W$ be any zero set in $\nu X-Z^{*}$. Take $f \in C^{b}$ such that $0 \leq \bar{f} \leq 1, \bar{f}=1$ on $W$ and $\bar{f}=0$ on $Z^{*}$. Since $m\left(X-Z_{d}\right)=0$ and since $f=0$ on $Z_{d}$, we have that $\bar{m}(W) \leq$ $\int_{\nu X} \bar{f} d \bar{m}=\bar{\phi}(\bar{f})=\int_{X} f d m=0$. Thus $\bar{m}(W)=0$ for every zero set $W \subset \nu X-Z^{*}$. The regularity of $\bar{m}$ now implies $\bar{m}\left(\nu X-Z^{*}\right)=0$ so that $G \subset Z^{*}$ as asserted.

We now claim that $\bar{g}_{n} \downarrow 0$ pointwise on $G$ (which implies that $\bar{f}_{i} \downarrow 0$ on G.) If this is not so, then there is an $x_{0} \in G$ and an $a>0$ such that $\bar{g}_{n}\left(x_{0}\right) \geq a$ for all $n$. Define $Z=\bigcap_{n-1}^{\infty}\left\{x \in \nu X: \bar{g}_{n}(x) \geq \alpha\right\}$. Then $Z \cap Z^{*}$ is a zero set in $\nu X$ with $x_{0} \in Z \cap Z^{*}$. Since $x_{0} \in \nu X$, we have that $X \cap Z \cap Z^{*} \neq \varnothing$. Let $x \in X$ $\cap Z \cap Z^{*}$. Since $Z_{d}=X \cap Z^{*}$, it follows that $x \in Z_{d} \cap Z^{*}$ which implies that $g_{n}(x) \geq a>0$ for all $n$. This contradicts the fact that $g_{n} \downarrow 0$ pointwise on $Z_{d}$. The proof is complete.

The following theorem summarizes the main facts.

Theorem 9.5. Let $X$ be a completely regular Hausdorff space (a D-space), and let $M_{s c}\left(M_{c}\right)$ be endowed with the topology of uniform convergence on the pointwise bounded and equicontinuous sets in $C$. Then the following bold:

1. $M_{s c}\left(M_{c}\right)$ is complete.

2. $L$ is dense in $M_{s c}\left(M_{c}\right)$.

3. The dual of $M_{s c}\left(M_{c}\right)$ is $C$.

4. The restriction of the topology of $M_{s c}\left(M_{c}\right)$ to $X$ is the original topology of $X$.

Proof. Both 1 and 2 are immediate from Theorem 9.4. That 3 holds is a consequence of Proposition 6.1 and the fact that the completion of a locally convex space has the same completion as the original space. Property 4 is a restatement of Proposition 6.2.

As a consequence of Theorem 9.5, we obtain the following result of Shirota. (See [4, p. 229].)

Theorem 9.6. Let $X$ be a D-space. Then $X$ is realcompact if and only if $X$ is complete for some uniform structure compatible with the topology of $X$.

Proof. The points in $\nu X$ may be identified with the ring homomorphisms of $C$ into $R$. These are nonnegative so that $\nu X$ is contained in $M_{c}$ by Proposition 4.4. It is not hard to verify that $\nu X$ is closed in $M_{c}$ for the topology of uniform

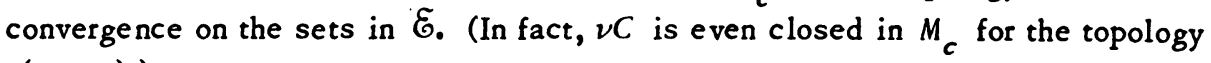
$\sigma\left(M_{c}, C\right)$.) Hence if $X=\nu X$, then, by Theorem 9.5, $X$ is complete for the uniformity induced on $X$ from $M_{c}$. On the other hand, if $X$ is complete for some 
uniformity, then it is complete for the finest uniformity compatible with $X$ [4, p. 231]. Since this is the uniformity generated by the family of all continuous pseudometrics on $X$, it is clear that it is the uniformity induced by $e$ on $X$. Hence $X$ is closed in $M_{c}$. The proof will be complete if we can show that $X$ is dense in $\nu X$ relative to the topology $e$ on $M_{c}$.

In order to see that $X$ is dense in $\nu X$, fix $x_{0} \in \nu X$ and $B \in \mathcal{G}$. It is enough to show that $X \cap\left(x_{0}+B^{\circ}\right) \neq \varnothing$, or, what is equivalent, that $p_{B}\left(x-x_{0}\right) \leq 1$ for some $x \in X$ (where $p_{B}$ is the Minkowski gauge of $B^{\circ}$ ). Define $f(x)=p_{B}\left(x-x_{0}\right)$ for all $x \in \nu X$, and set $Z=\{x \in \nu X: f(x) \leq 1\}$. Then since $f \in C(\nu X), Z$ is a zero set in $\nu X$. This means that $Z \cap X \neq \varnothing$, and the proof is complete.

We can now prove the following analogue of Granirer's result (Corollary 8.8).

Corollary 9.7. Let $X$ be completely regular Hausdorff (a D-space).

1. If $B$ is a pointwise bounded and equicontinuous subset of $C$, then $B$ is relatively $\sigma\left(C, M_{s c}\right)$-compact $\left(\sigma\left(C, M_{c}\right)\right.$-compact $)$.

2. If $X$ is not a D-space, then there is a pointwise bounded equicontinuous set $B$ in $C$ which is not $\sigma\left(C, M_{c}\right)$-compact.

Proof. 1. This is an immediate consequence of Theorem 9.5 and the BanachAlaoglu theorem.

2. If $X$ is not a $D$-space, then $M_{s c} \neq M_{c}$. Let $M_{c}$ have the topology of uniform convergence on the sets in $\mathcal{E}_{\text {. Then }} M_{s c}$ is a proper closed subspace of $M_{c}$ by Theorem 9.5. By the Hahn-Banach theorem, there is a continuous linear functional on $M_{c}$ which vanishes on $X$. Hence the dual of $M_{c}$ is strictly larger than $C$. The result now follows by the Mackey-Arens theorem.

Remarks. 1. If $X$ is compact (or pseudocompact), then Theorem 9.4 reduces to the Katětov-Ptăk theorem.

2. If $X$ is discrete and nonmeasurable, then $M_{c}=L$.

Theorem 9.8. Let $X$ be a completely regular Hausdorff space and let $M_{c}$ bave the topology of uniform convergence on the pointwise bounded and equicontinuous subsets of $C$. Then the following are equivalent:

1. $X$ is finite.

2. $M_{c}$ is normable.

3. $M_{c}$ is metrizable.

4. $(L, e)$ is metrizable.

Proof. $(1 \Rightarrow 2)$ This is obvious.

$(2 \Rightarrow 3)$ This is obvious.

$(3 \Rightarrow 4)$ This is obvious.

$(4 \Rightarrow 1)$ Take a sequence $\left\{B_{n}\right\} \subset \mathcal{E}$ such that $B_{n} \subset B_{n+1}$ and such that 
$\left\{B_{n}^{\circ}\right\}$ form a local base at zero for $e$. Also assume that $B_{n}^{\circ \circ}=B_{n}$ which is possible by Proposition 5.1 and the bipolar theorem. Then if $B \in \mathcal{E}$, there is an $n$ such that $B \subset B_{n}^{\circ \circ}=B_{n}$. Define $B_{n}^{b}=B_{n} \cap\left\{f \in C^{b}:\|f\|_{X} \leq n\right\}$. It is clear that if $B \in \mathcal{E}^{b}$, then $B^{n} \subset B_{n}^{b}$ for some $n$. Hence $\left\{B_{n}^{b_{0}}\right\}$ is a local base at zero for $e^{b}$. This means that $e^{b}$ is metrizable. Hence $\left(L, e^{b}\right)$ is a Mackey space. Hence by Theorem $8.9, X$ is discrete. If $X$ is not finite, let $\left\{x_{n}\right\} \subset X$ be an infinite subset. For each $n$, take a number $\alpha_{n}$ such that $\sup \left\{/\left(x_{n}\right): f \in B_{n}\right\}<$ $a_{n}$. Finally, let $f$ be any real-valued function on $X$ such that $f\left(x_{n}\right)^{n}=a_{n}$ for all $n$. Then $\{f\} \in \mathcal{E}$ but $f \notin B_{n}$ for any $n$. This is a contradiction, and the proof is complete.

10. Completion of $L$ for the Mackey topology of $(L, C)$. Let $m$ denote the Mackey topology on $L$ for the pair $(L, C)$, and let $\hat{L}^{m}$ denote the completion of this space. We will investigate the space $\hat{L}^{m}$ in this section. We will first develop some facts which will be very useful for this purpose. Proposition 10.2 seems to be of some interest in its own right.

Lemma 10.1. Let $B$ be a compact, convex and balanced subset of $R^{X}$ with its product topology. If $\left\{f_{n}\right\}$ is a sequence of elements of $B$ and if $\left\{a_{n}\right\}$ is a sequence of numbers with $\sum_{n=1}^{\infty}\left|a_{n}\right| \leq 1$, then the function $g=\sum_{n=1}^{\infty} a_{n} l_{n}$ belongs to $B$.

Proof. Since $B$ is pointwise bounded, for each $x \in X, \sup \{|f(x)|: x \in X\}$ is finite so that $g \in R^{X}$. Define $g_{n}=\sum_{k=1}^{n} a_{k} f_{k}$. Since $\Sigma^{\infty}\left|a_{n}\right| \leq 1$ and since $B$ is convex and balanced, $g_{n} \in B$ for all $n \in N$. Furthermore, $g_{n} \rightarrow g$ pointwise. Since $B$ is closed, it follows that $g \in B$. The proof is complete.

Proposition 10.2. Let $B$ be a compact convex and balanced subset of $R^{X}$ with its product topology. If each function in $B$ is uniformly bounded, then the family $B$ is uniformly bounded. (That is, $\sup \left\{\|f\|_{X}: f \in B\right\}<\infty$.)

Proof. Assume that the proposition is false, and let $\left\{f_{n}\right\}$ be a sequence in $B$ with $\left\|f_{n}\right\|_{X} \uparrow+\infty$ as $n \rightarrow \infty$. For each $x \in X$ define a function $\beta_{x}: N \rightarrow R$ (where $N$ is the set of positive integers) by $\beta_{x}(n)=f_{n}(x)$. Since $B$ is pointwise bounded, $\left\{\beta_{x}: n \in N\right\} \subset l^{\infty}$.

Now let $a=\left\{a_{n}\right\} \in l^{1}$ with $\|\alpha\|_{1} \leq 1$, and define $f_{\alpha}=\sum_{n=1}^{\infty} a_{n} f_{n}$. Then $f_{a}$ $\epsilon B$ by Lemma 10.1. Also, regarding $l^{\infty}$ as the Banach dual of $l^{1}$, we see that, for all $x \in X,\left|\beta_{x}(\alpha)\right|=\left|\Sigma_{n=1}^{\infty} a_{n} f_{n}(x)\right|=\left|f_{a}(x)\right| \leq\left\|f_{a}\right\|_{X}$. Thus it follows that $\left\{\beta_{x}: x \in X\right\}$ is a pointwise bounded family of continuous linear functionals on $l^{1}$. By the Banach-Steinhaus theorem, there is a number $M$ such that $\left\|\beta_{x}\right\|_{\infty} \leq M$ for all $x \in X$. But this implies that $\left|f_{n}(x)\right| \leq M$ for all $x \in X$ and all $n \in N$. Hence $\left\|f_{n}\right\|_{X} \leq M$ for all $n \in N$ which contradicts the fact that $\left\|f_{n}\right\|_{X} \uparrow \infty$. The proof is complete. 
Remark. It is easy to see that the hypothesis that $B$ is convex and balanced cannot be omitted in the above theorem. Indeed take $X=N$ and $B=\{0\} \cup$ $\left\{n f_{n}: n \in N\right\}$ where $f_{n}$ is the characteristic function of $\{n\}$. Then $B$ is compact in $R^{N}$ and each function in $B$ is uniformly bounded, although $B$ itself is not uniformly bounded.

The following is a result of A. Grothendieck. A proof may be found in [17, p. 325].

Theorem 10.3. Let $X$ be a compact Hausdorff space and let $A \subset C(X)$. Then $A$ is relatively weakly compact if and only if $A$ is relatively $\sigma(C, L)$-compact and uniformly bounded.

Proposition 10.4. (a) $\hat{L}^{m}$ is a subspace of $M_{s c^{\circ}}$

(b) If $m \in M$ and if $m$ bas compact support, then $m \in \hat{L}^{m}$.

Proof. (a) Let $\phi \in \hat{L}^{m}$. Then by Proposition 5.1 and the completion theorem, $\phi$ is $\sigma(C, L)$-continuous on each $B \in \mathcal{E}$. Hence $\phi$ is in the completion of $(L, e)$. It then follows by Theorem 9.4 that $\phi \in M_{s c^{\circ}}$

(b) If $m$ has compact support in $X$, then $m$ is net-additive and, hence, a regular Borel measure by Proposition 1.8. Let $G$ denote the support of $m$. Take any set $A \subset C$ which is $\sigma(C, L)$-compact, convex and balanced. By the completion theorem, we must show that if $\left\{f_{i}\right\} \subset A$ is any net with $f_{i} \rightarrow 0$ pointwise on $X$, then $\int_{X} f_{i} d m \rightarrow 0$.

Assume that there is a net $\left\{f_{i}\right\} \subset A$ such that $f_{i} \rightarrow 0$ pointwise on $X$ while $\left|\int_{X} f_{i} d m\right| \geq \alpha>0$ for all $i$. Now let $A_{G}$ denote the set of functions formed by restricting the functions in $A$ to $G$. Then $A_{G}$ is a $\sigma(C(G), L(G)$ )-compact, convex and balanced subset of $C(G)$. Since $G$ is compact, each function in $A_{G}$ is uniformly bounded. Hence by Proposition 10.2 the family $A_{G}$ is uniformly bounded. Hence by Theorem $10.3, A_{G}$ is a weakly compact subset of $C(G)$. Let $f_{i}^{*}$ denote the restriction of $f_{i}$ to $G$. Since $A_{G}$ is weakly compact there is a subnet $\left\{g_{j}^{*}\right\}$ of $\left\{f_{j}^{*}\right\}$ such that $g_{j}^{*} \rightarrow 0$ weakly on $G$. Since $m$ is supported on $G, m$ belongs to the Banach dual of $C(G)$. Hence $\int_{X} g_{j} d m \rightarrow 0$. This contradicts the assumption that $\left|\int_{X} f_{i} d m\right| \geq a>0$ for all $i$. The proof is complete.

Theorem 10.5. Let $X$ be realcompact. Then the completion of $L$ for the Mackey topology of the pair $(L, C)$ is the space $M_{c}$ with the Mackey topology of the pair $\left(M_{c}, C\right)$.

Proof. It is immediate from Proposition 10.4 that $\hat{L}^{m}=M_{c}$. Furthermore, the completion of a Mackey space is a Mackey space. (See [17, p. 262].) The proof is complete.

The following is a generalization of Grothendieck's result (Theorem 10.3). 
Theorem 10.6. Let $X$ be realcompact and let $A \subset C$ be convex and balanced. Then $A$ is relatively $\sigma\left(C, M_{c}\right)$-compact if and only if it is relatively $\sigma(C, L)$ compact.

Proof. The completion of a locally convex space has the same equicontinuous sets in the dual space. (See $[17, \mathrm{p} .261]$.) Hence if $A \subset C$ is $\sigma(C, L)$-compact, convex and balanced (and hence equicontinuous for the Mackey topology of the pair $(L, C)$ ), then $A$ is equicontinuous for the Mackey topology for the pair $\left(M_{c}, C\right)$ by Theorem 10.5. Hence $A^{\circ 0}$ is $\sigma\left(C, M_{c}\right)$-compact by the Alaoglu theorem. Hence $A$ is relatively $\sigma\left(C, M_{c}\right)$-compact. The proof is complete.

It seems reasonable to conjecture that for an arbitrary completely regular Hausdorff space, the completion of $L$ for the Mackey topology of the pair $(L, C)$ is the space $M_{\dot{s}}$. This conjecture is supported by Theorem 10.5. Furthermore, it has been proved by Ptăk [22, p. 187] that the conjecture holds if $X$ is pseudocompact. The author hopes to return to this question in a future note. The following consequence of Theorem 10.5 is of some interest.

Theorem 10.7. Let $X$ be completely regular Hausdorff, and let $M_{c}$ be endowed with the Mackey topology for the pair $\left(M_{c}, C\right)$. Then $M_{c}$ is complete and $L$ is dense in $M_{c}$.

Proof. For $f \in C$, let $\bar{f}$ denote its unique continuous extension to $\nu X$. Define a map $T: M_{c}(X) \rightarrow M_{c}(\nu X)$ by $T(m)=\bar{m}$ where $\bar{m}$ is the unique element in $M_{c}(\nu X)$ such that $\int_{X} f d m=\int_{\nu X} \bar{f} d \bar{m}$ for all $f \in C(X)$. It is easily verified that $T$ is a topological isomorphism when $M_{c}(X)$ has the weak topology $\sigma\left(M_{c}(X), C(X)\right)$ and $M_{c}(\nu X)$ has the weak topology $\sigma\left(M_{c}(\nu X), C(\nu X)\right)$. Hence $T$ is a topological isomorphism when $M_{c}(X)$ and $M_{c}(\nu X)$ have their respective Mackey topologies. Since $M_{c}(\nu X)$ is complete for its Mackey topology by The orem 10.5 and since $T^{-1}$ is a uniform space isomorphism, $M_{c}(X)$ is complete for its Mackey topology. It is clear that $L$ is dense in $M_{c^{*}}$. The proof is complete.

The final question that arises is what is the completion (denoted by $\hat{L}^{b m}$ ) of $L$ for the Mackey topology of the pair $\left(L, C^{b}\right)$. We will present several results related to this question.

Proposition 10.8. (a) $\hat{L}^{b m} \subset M_{s}$.

(b) $\hat{L}^{b m}$ contains the subspace of $M$ consisting of those set functions which have compact support.

Proof. (a) Since if $B \in \mathcal{E}^{b}$, then $B^{D \circ} \in \mathcal{E}^{b}$, this is immediate from Theorem 9.5 and the completion the orem.

(b) Since $\hat{L}^{m} \subset \hat{L}^{b m}$ by the completion theorem, this is immediate from Proposition 10.4. 
The general representation of $\hat{L}^{b m}$ is unknown to the author at the present time, but he hopes to return to this question in the fut ure. The following result is analogous to Theorem 10.7. It follows from the fact that $C^{b}$ is a Banach space (see [26, Chapter VI, $\$ 1$, Corollary 2 to Proposition 1]). We will include a proof for completeness.

Theorem 10.10. Let $X$ be completely regular Hausdorff, and let $M$ be endowed with the Mackey topology for the pair $\left(M, C^{b}\right)$. Then $M$ is complete and $L$ is dense in $M$.

Proof. For $f \in C^{b}(X)$, let $\bar{f}$ denote its unique continuous extension to $X$. Define a map $T: M(X) \rightarrow M(\beta X)$ by $T(m)=\bar{m}$ where $\bar{m}$ is the unique element in $M(\beta X)$ such that $\int_{X} f d m=\int_{X} \bar{f} d \bar{m}$ for all $f \in C^{b}(X)$. Then $T$ is a topological isomorphism for the weak topologies. Hence $T$ is a topological isomorphism for the Mackey topologies. Since $M(\beta X)=M_{c}(\beta X), M(\beta X)$ is complete for the Mackey topology by Theorem 10.5. Since $T^{-1}$ is a uniform space isomorphism, it follows that $M(X)$ is complete for its Mackey topology. It is clear that $L$ is dense in $M$. The proof is complete.

\section{BIBLIOGRAPHY}

1. A. D. Alexandrov, Additive set functions in abstract spaces, Mat. Sb. 8(50) (1940), 307-348; 9(51) (1941), 563-628. MR 2, 315; 3, 207.

2. R. Arens and J. Eells, On embedding uniform and topological spaces, Pacific J. Math. 6 (1956), 397-403. MR 18, 406.

3. R. M. Dudley, Convergence of Baire measures, Studia Math. 27 (1966), 251-268. MR 34 \#598.

4. L. Gillman and M. Jerison, Rings of continuous functions, University Series in Higher Math., Van Nostrand, Princeton, N. J., 1960. MR 22 \#6994.

5. G. Gould and M. Mahowald, Measures on completely regular spaces, J. London Math. Soc. 37 (1962), 103-111. MR 27 \#6122.

6. E. Granirer, On Baire measures on D-topological spaces, Fund. Math. 60 (1967), 1-22. MR $34 \# 8165$.

7. E. Hewitt, Linear functionals on spaces of continuous functions, Fund. Math. 37 (1950), 161-189. MR 13, 147.

8. M. Katětov, Measures in fully normal spaces, Fund. Math. 38 (1951), 73-84. MR 14, 27.

9. - On a category of spaces, General Topology and its Relations to Modern Analysis (Proc. Sympos., Prague, 1961), Academic Press, New York; Publ. House Czech. Acad. Sci., Prague, 1962, pp. 226-229. MR $32 \# 4644$.

10. - On certain projectively generated continuity structures, Celebrazioni archimedee de secolo, Simposio di topologia, 1964, pp. 47-50.

11. - Projectively generated continuity structures: A correction, Comment. Math. Univ. Carolinae 6 (1965), 251-255. MR 36 \#2110. 
12. R. B. Kirk, Measures in topological spaces and B-compactness, Nederl. Akad. Wetensch. Proc. Ser. A 72 = Indag. Math. 31 (1969), 172-183. MR 39 \#410.

13. - Locally compact, B-compact spaces, Nederl. Akad. Wetensch. Proc. Ser. A 72 = Indag. Math. 31 (1969), 333-344. MR 41 \#9201.

14. - Algebras of bounded real-valued functions, Nederl. Akad. Wetensch. Proc. Ser. A 75 = Indag. Math. 34 (1972), 443-463.

15. - - A note on the Mackey topology for $\left(C^{b}(X)^{*}, C^{b}(X)\right)$, Pacific J. Math. (to appear).

16. J. D. Knowles, Measures in topological spaces, Proc. London Math. Soc. 17 (1967), 139-156. MR 34 \#4441.

17. G. Köthe, Topologische linear Räume. I, Die Grundlehren der math. Wissenschaften, Band 107, Springer-Verlag, Berlin, 1960; English transl., Die Grundlehren der math.

Wissenschaften, Band 159, Springer-Verlag, New York, 1969. MR 24 \#A411; 40 \#1750.

18. W. Moran, The additivity of measures on completely regular spaces, J. London. Math. Soc. 43 (1968), 633-639. MR 37 \#4225.

19. - - Measures on metacompact spaces, Proc. London Math. Soc. 20 (1970), 507-524.

20. E. Marczewski and R. Sikorski, Measures in non-separable metric spaces, Colloq. Math. 1 (1948), 133-139. MR 10, 23.

21. J. S. Pym, Positive functionals, additivity and supports, J. London Math. Soc. 39 (1964), 391-399. MR 29 \#2641.

22. V. Ptăk, Algebraic extensions of topological spaces, Contributions to Extension Theory of Topological Structures, (Proc. Sympos., Berlin, 1967), Deutsch. Verlag Wissenschaften, Berlin, 1969, pp. 179-188. MR 40 \#681.

23. D. A. Raukov, Free locally convex spaces for uniform spaces, Mat. Sb. 63 (105) (1964), 582-590. (Russian) MR 28 \#5320.

24. F. D. Sentilles, and R. F. Wheeler, Additivity of functionals and the strict topology (unpublished).

25. V. S. Varadarajan, Measures on topological spaces, Mat. Sb. 55 (97) (1961), 35-100; English transl., Amer. Math. Soc. Transl. (2) 48 (1965), 161-228. MR 26 \#6342.

26. A. P. Robertson and W. J. Robertson, Topological vector spaces, Cambridge Univ. Press, New York, 1964. MR 28 \#5318.

DEPARTMENT OF MATHEMATICS, SOUTHERN ILLINOIS UNIVERSITY, CARBONDALE, ILLINOIS 62901 